\title{
Thermochemical Properties of Halides and Halohydrides of Silicon and Carbon
}

\author{
Pitsiri Sukkaew, Lars Ojamäe, Olle Kordina, Erik Janzén and Örjan Danielsson
}

\author{
Linköping University Post Print
}

Tweet

N.B.: When citing this work, cite the original article.

Original Publication:

Pitsiri Sukkaew, Lars Ojamäe, Olle Kordina, Erik Janzén and Örjan Danielsson, Thermochemical Properties of Halides and Halohydrides of Silicon and Carbon, 2016, ECS Journal of Solid State Science and Technology, (5), 2, P27-P35.

http://dx.doi.org/10.1149/2.0081602jss

Copyright: (C) The Author(s) 2015. Published by ECS. This is an open access article distributed under the terms of the Creative Commons Attribution 4.0 License http://www.electrochem.org/

Postprint available at: Linköping University Electronic Press http://urn.kb.se/resolve?urn=urn:nbn:se:liu:diva-124117 


\title{
Thermochemical Properties of Halides and Halohydrides of Silicon and Carbon
}

\author{
Pitsiri Sukkaew, ${ }^{\mathrm{Z}}$ Lars Ojamäe, Olof Kordina, Erik Janzén, and Örjan Danielsson ${ }^{\mathrm{z}}$
}

Department of Physics, Chemistry and Biology, Linköping University, Linköping, Sweden

\begin{abstract}
Atomization energies, enthalpies of formation, entropies as well as heat capacities of the $\mathrm{SiH}_{\mathrm{n}} \mathrm{X}_{\mathrm{m}}$ and $\mathrm{CH}_{\mathrm{n}} \mathrm{X}_{\mathrm{m}}$ systems, with $\mathrm{X}$ being $\mathrm{F}, \mathrm{Cl}$ and $\mathrm{Br}$, have been studied using quantum chemical calculations. The Gaussian-4 theory (G4) and Weizman-1 theory as modified by Barnes et al. 2009 (W1RO) have been applied in the calculations of the electronic, zero point and thermal energies. The effects of low-lying electronically excited states due to spin orbit coupling were included for all atoms and diatomic species by mean of the electronic partition functions derived from the experimental or computational energy splittings. The atomization energies, enthalpies of formation, entropies and heat capacities derived from both methods were observed to be reliable. The thermochemical properties in the temperature range of $298-2500 \mathrm{~K}$ are provided in the form of 7-coefficient NASA polynomials.

(C) The Author(s) 2015. Published by ECS. This is an open access article distributed under the terms of the Creative Commons Attribution 4.0 License (CC BY, http://creativecommons.org/licenses/by/4.0/), which permits unrestricted reuse of the work in any medium, provided the original work is properly cited. [DOI: 10.1149/2.0081602jss] All rights reserved.
\end{abstract}

Manuscript submitted September 8, 2015; revised manuscript received October 22, 2015. Published November 10, 2015.

Silicon carbide $(\mathrm{SiC})$ is an attractive material for power electronics. The characteristics of having wide bandgap, high thermal conductivity, high blocking voltage and switching frequencies have made it superior for applications at high temperatures, frequencies and voltages. ${ }^{1}$ To this end, it is vital to achieve high material quality as well as manufacturing process reliability and efficiency. In chemical vapor deposition (CVD), a widely used fabrication method for SiC layers, this means suppressing the formation of Si clusters and parasitic depositions which are known to introduce defects and degrade the growing layers, along with shortening the lifetime of reactor components. ${ }^{2-4}$ Halogenated gases have been utilized to reduce the cluster formation by breaking the $\mathrm{Si}-\mathrm{Si}$ bonds in the clusters and forming the stronger Si-halogen bonds and consequently introduce a new parameter into the process, namely the halogen/Si ratio. This ratio has shown impacts on not only the cluster formation and parasitic growth, but also on the growth rates as well as defect formations. ${ }^{4,5}$

In complex processes such as CVD, computational modeling has become essential in research as well as process development and design. Modeling accuracy and correctness depend crucially on the quality of the kinetics and thermochemistry data input. Thermochemistry data of some halides and halohydrides of $\mathrm{Si}$ and $\mathrm{C}$ are provided in databases, ${ }^{6-8}$ review, ${ }^{9}$ as well as experimental ${ }^{10-16}$ and theoretical studies. ${ }^{17-32}$ Nevertheless, inconsistency derived from various means of achieving data along with many data mismatches have rendered it far from complete. Here we present thermochemical properties from quantum chemical calculations of a complete set of halides and halohydrides of $\mathrm{Si}$ and $\mathrm{C}$, namely $\mathrm{SiX}_{\mathrm{n}} \mathrm{H}_{\mathrm{m}}$ and $\mathrm{CX}_{\mathrm{n}} \mathrm{H}_{\mathrm{m}}$ with $\mathrm{X}=\mathrm{F}, \mathrm{Cl}$ and $\mathrm{Br}$, and $\mathrm{n}+\mathrm{m} \leq 4$, covering the temperature range of $298-2500 \mathrm{~K}$. The study range accommodates applications such as CVD process, surface etching of semiconductors, combustion, as well as fundamental studies. The quantum chemistry composite methods of Gaussian-4 theory $(\mathrm{G} 4)^{33}$ are utilized and compared to the Weizmann-1 theory ${ }^{34,35}$ as modified by Barnes et al. $2009^{36}$ (W1RO).

\section{Computational Methods}

All quantum chemical calculations were carried out using Gaussian 09. ${ }^{37}$ The G4 and W1RO methods are described in detail in Ref. 33 and Ref. 34-36. Both methods are designed to achieve the total energy from its additives derived from different levels of theories. The G4 uses the B3LYP/6-31G(2df,p) level in the geometry optimization as well as the harmonic frequency calculation. The frequencies are then scaled using a factor of $0.9854-$ a factor optimized for the zero point energy (ZPE) calculated at this level. The electronic energy is composed of the following terms: the Hartree-Fock (HF) limit energy, the electron correlation energy, the higher level empirical corrections (HLCs) and for atoms the spin-orbit corrections. The inclusion of HLCs significantly helps reducing the computational cost while maintaining the accuracy within acceptable range. The theory is attractive to applications on halide systems due to many improvements over its previous version, Gaussian-3 (G3) ${ }^{38,39}$ such as an addition of a complete basis set limit (CBS) extrapolation of the HF energy, the increase of the $d$-polarization sets on the first- and second-row atoms with the exponents reoptimized for the latter, and an improvement in the HLCs on radicals.

W1RO theory is based on the geometry optimized at the B3LYP/cc$\mathrm{pVTZ}(+\mathrm{d})$ level, where $(+\mathrm{d})$ refers to the addition of a tight $d$ function in the presence of second-row atoms. The harmonic frequencies are obtained from the same level and scaled with a factor of 0.985 - also a scaling factor for ZPE. The electronic energy is composed of the CBS values of the self-consistent field energy (SCF), the coupled cluster with all single and double substitutions (CCSD) and its perturbative triple excitation correction $(\mathrm{T})$ together with the core correlation and scalar relativistic energy following the method modified by Barnes et al., ${ }^{36}$ and the spin orbit corrections for atoms. Both close-shell and open-shell molecules are treated similarly using the spin-restricted theory, and henceforth referred to as W1RO following Barnes et al. ${ }^{36}$ Apart from the spin orbit corrections and extrapolations, the W1RO contains no empirical corrections.

The electronic energy, ZPE and thermal energy at $298 \mathrm{~K}$ from scaled harmonic frequencies contribute to the energy at $0 \mathrm{~K}\left(\mathrm{H}^{0}\right)$ and the enthalpies at $298 \mathrm{~K}\left(\mathrm{H}^{298}\right)$. The atomization energies at $0 \mathrm{~K}\left(\Sigma \mathrm{D}_{0}\right.$ $(0 \mathrm{~K}))$ and enthalpies of formation $\left(\Delta \mathrm{H}_{f}^{\circ}\right)$ at $0 \mathrm{~K}$ and $298 \mathrm{~K}$ are derived from the following equations,

$$
\begin{gathered}
\Sigma \mathrm{D}_{0}(0 \mathrm{~K})=\Sigma_{\text {atom }}\left[\mathrm{H}^{0}\right]_{\text {atoms }}-\mathrm{H}_{\text {molecule }}^{0}, \\
\Delta \mathrm{H}_{f}^{\circ}(0 \mathrm{~K})=\Sigma_{\text {atom }}\left[\Delta \mathrm{H}_{f}^{\circ}(0 \mathrm{~K})\right]_{\text {atoms }}-\Sigma \mathrm{D}_{0}(0 \mathrm{~K}), \\
\Delta \mathrm{H}_{f}^{\circ}(298 \mathrm{~K})=\Delta \mathrm{H}_{f}^{\circ}(0 \mathrm{~K})+\left(\mathrm{H}^{298}-\mathrm{H}^{0}\right)_{\text {molecule }} \\
-\Sigma_{\text {atom }}\left[\mathrm{H}^{298}-\mathrm{H}^{0}\right]_{\text {atom }} .
\end{gathered}
$$

The atomic $\Delta \mathrm{H}_{f}^{\circ}(0 \mathrm{~K})$ and the atomic thermal enthalpy corrections at $298 \mathrm{~K}\left(\mathrm{H}^{298}-\mathrm{H}^{0}\right)$ used in the study are shown in Table I.

The $\Delta \mathrm{H}_{f}^{\circ}(298 \mathrm{~K})$ and the thermal enthalpy corrections at temperature $\mathrm{T}\left(\mathrm{H}^{\mathrm{T}}-\mathrm{H}^{298}\right)$ provide the absolute enthalpy, $\Delta \mathrm{H}^{\mathrm{T}} \equiv \Delta \mathrm{H}_{f}^{\circ}$ $(298 \mathrm{~K})+\left[\mathrm{H}^{\mathrm{T}}-\mathrm{H}^{298}\right]$. In contrast to $\left(\mathrm{H}^{298}-\mathrm{H}^{0}\right)$ and $\mathrm{ZPEs}$, vibrational contributions to $\left(\mathrm{H}^{\mathrm{T}}-\mathrm{H}^{298}\right)$, entropies $\left(\mathrm{S}^{\mathrm{T}}\right)$ and heat capacities $\left(\mathrm{C}_{\mathrm{p}}^{\mathrm{T}}\right)$ were calculated based on the unscaled harmonic frequencies rather than the ZPE scaled harmonic frequencies. The G4 and W1RO calculated $\Delta \mathrm{H}^{\mathrm{T}}, \mathrm{S}^{\mathrm{T}}$ and $\mathrm{C}_{\mathrm{p}}^{\mathrm{T}}$ are fitted into 2 sets of 7-coefficient NASA 
Table I. Atomic $\Delta \mathrm{H}_{f}^{\circ}(0 \mathrm{~K})$ and thermal corrections to enthalpy at $298 \mathrm{~K}\left(\mathrm{H}^{298}-\mathrm{H}^{0}\right)$ in $\mathrm{kJ} \cdot \mathrm{mol}^{-1}$.

\begin{tabular}{|c|c|c|c|c|c|}
\hline & $\Delta \mathrm{H}_{f}^{\circ}(0 \mathrm{~K})$ & $\mathrm{H}^{298}-\mathrm{H}^{0}$ & & $\Delta \mathrm{H}_{f}^{\circ}(0 \mathrm{~K})$ & $\mathrm{H}^{298}-\mathrm{H}^{0}$ \\
\hline $\mathrm{Si}$ & $\begin{array}{l}446 \pm 8^{\mathrm{a}} \\
445.67 \pm 8.00^{\mathrm{b}} \\
448.32 \pm 0.84^{\mathrm{c}} \\
448.5 \pm 0.8^{\mathrm{d}} \\
452.3 \pm 2^{\mathrm{e}}\end{array}$ & $\begin{array}{l}3.218^{\mathrm{a}} \\
3.217^{\mathrm{b}, \mathrm{c}}\end{array}$ & $\mathrm{C}$ & $\begin{array}{l}711.19 \pm 0.46^{\mathrm{a}} \\
711.19 \pm 0.45^{\mathrm{b}} \\
711.198 \pm 0.45^{\mathrm{c}}\end{array}$ & $\begin{array}{l}1.051^{\mathrm{a}} \\
1.050^{\mathrm{b}} \\
1.054^{\mathrm{c}}\end{array}$ \\
\hline $\mathrm{H}$ & $\begin{array}{l}216.035 \pm 0.006^{\mathrm{a}} \\
216.04 \pm 0.01^{\mathrm{b}} \\
216.034 \pm 0.001^{\mathrm{c}}\end{array}$ & $4.234^{\mathrm{a}-\mathrm{c}}$ & $\mathrm{F}$ & $\begin{array}{l}77.28 \pm 0.30^{\mathrm{a}} \\
77.27 \pm 0.30^{\mathrm{b}} \\
77.274 \pm 0.058^{\mathrm{c}}\end{array}$ & $4.413^{\mathrm{a}-\mathrm{c}}$ \\
\hline $\mathrm{Cl}$ & $\begin{array}{l}119.621 \pm 0.006^{\mathrm{a}} \\
119.63 \pm 0.01^{\mathrm{b}} \\
119.620 \pm 0.002^{\mathrm{c}}\end{array}$ & $4.591^{\mathrm{a}-\mathrm{c}}$ & $\mathrm{Br}$ & $\begin{array}{l}117.92 \pm 0.06^{\mathrm{a}} \\
117.93 \pm 0.12^{\mathrm{b}} \\
117.915 \pm 0.06^{\mathrm{c}}\end{array}$ & $\begin{array}{l}12.255^{\mathrm{a}} \\
12.260^{\mathrm{b}, \mathrm{c}}\end{array}$ \\
\hline
\end{tabular}

${ }^{a}$ NIST-JANAF Database, 1998 ; ${ }^{b}$ NIST-CCCBDB Database ${ }^{40} ;{ }^{c}$ Goos-Burcat-Ruscic Thermochemical Database for Combustion ${ }^{7}$; ${ }^{\mathrm{d}}$ Karton and Martin,

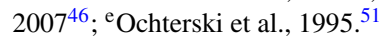

polynomials, corresponding to the temperature ranges of 298.15$1000 \mathrm{~K}$ and $1000-2500 \mathrm{~K}$.

\section{Results and Discussion}

Atomization energies $\left(\Sigma D_{0}\right)$.- The electronic energies and ZPEs calculated using the G4 and W1RO methods are provided in supplementary material. The atomization energies $\left(\Sigma \mathrm{D}_{0}(0 \mathrm{~K})\right)$ derived from the G4 and W1RO methods are presented in Table II in comparison to the databases/experiments and theoretical calculations from literature. In contrast to the G4 method, the W1RO has been designed only for atoms up to the second-row of the periodic system. Therefore this method is only used here for the $\mathrm{F}$ - and $\mathrm{Cl}$-systems.

It is clear from Table II that the database/experimental atomization energies for many species are inconsistent. On the other hand, good agreement is observed between our calculations and those derived from highly accurate computational methods reported by Feller et al., ${ }^{26}$ Feller and Dixon, ${ }^{28}$ Vasiliu et al., ${ }^{27}$ Karton et al., ${ }^{30}$ Tajti et al., ${ }^{31}$ Grant and Dixon ${ }^{32}$ and Klopper et al. ${ }^{29}$ It is apparent that large dis- crepancies of $\sim 15-40 \mathrm{~kJ} \mathrm{~mol}^{-1}$ exist between the theoretical and experimental results (NIST-JANAF ${ }^{6}$ and NIST-CCCBDB ${ }^{40}$ ) of $\mathrm{SiF}$, $\mathrm{SiF}_{2}, \mathrm{CHF}, \mathrm{SiH}_{3} \mathrm{~F}$ and $\mathrm{CH}_{3} \mathrm{~F}$ (NIST-CCCBDB only), accompanied by small to moderate discrepancies of $\sim 7-12 \mathrm{~kJ} \mathrm{~mol}^{-1}$ for SiH (NISTJANAF only), $\mathrm{CF}$ and $\mathrm{CF}_{2}$. Naturally, such discrepancies are observed in the corresponding $\Delta \mathrm{H}_{f}^{\circ}$ as shown later in Tables III and IV. The data from the active thermochemical table database (ATcT), ${ }^{20}$ on the other hand, are much more agreeable to the theory.

It is observed by comparing the $\mathrm{W} 1 \mathrm{RO}$ energies to the more expensive model of $\mathrm{W} 4$ theory ${ }^{30}$ that the deviations in $\mathrm{CF}_{4}$ and $\mathrm{CH}_{2} \mathrm{~F}_{2}$ are caused by the slow convergence in the CCSD and SCF energies with increased basis sets, while the deviations in $\mathrm{SiF}_{4}$ and $\mathrm{SiH}_{3} \mathrm{~F}$ are due to the core-correlation and scalar relativistic energies. This points out the necessity to consider calculation methods from higher levels if the results with higher accuracies are required.

On the other hand, the G4 theory is observed to depend strongly on the HLCs, which contributes $\sim 14-30 \mathrm{~kJ} \mathrm{~mol}^{-1}$ to the $\Sigma \mathrm{D}_{0}$ and helps to reduce computational costs tremendously. Compared to the more expensive methods, the G4 performs very nicely despite its low cost.

Table II. Atomization energies $\left(\Sigma D_{0}(0 \mathrm{~K})\right)$ in $\mathrm{kJ} \cdot \mathrm{mol}^{-1}$ derived from the $\mathrm{G} 4$ and W1RO methods in comparison to the databases and experiments from literature. Due to the method construction, the W1RO is applied only to the F- and Cl-systems.

\begin{tabular}{|c|c|c|c|c|}
\hline & \multicolumn{2}{|c|}{ This work } & \multicolumn{2}{|c|}{ Literature/Databases } \\
\hline & G4 & W1RO & Databases/Experiments & Theory \\
\hline $\mathrm{SiH}$ & 296.6 & 294.6 & $287.1 \pm 8.4^{\mathrm{a}}, 294.8^{\mathrm{b}}$ & $294.9 \pm 0.8^{\mathrm{e}}, 295.0 \pm 1.7^{\mathrm{h}}$ \\
\hline $\mathrm{SiF}$ & 589.7 & 587.7 & $545.2 \pm 12.6^{\mathrm{a}}, 550.0^{\mathrm{b}}$ & $585.3 \pm 1.7^{\mathrm{h}}$ \\
\hline $\mathrm{SiF}_{2}$ & 1235.6 & 1236.7 & $1187.6 \pm 12.6^{\mathrm{a}}$ & $1231.8 \pm 2.1^{\mathrm{h}}$ \\
\hline $\mathrm{SiF}_{3}$ & 1674.9 & 1678.2 & $1760.1 \pm 16.7^{\mathrm{a}}$ & \\
\hline $\mathrm{SiF}_{4}$ & 2364.0 & 2370.9 & $2364.5 \pm 0.84^{\mathrm{a}}, 2364.3^{\mathrm{b}}$ & $2367.8^{\mathrm{j}}$ \\
\hline $\mathrm{SiHF}$ & 897.1 & 897.2 & & \\
\hline $\mathrm{SiH}_{2} \mathrm{~F}$ & 1131.9 & 1135.7 & & $1136.2^{1}$ \\
\hline $\mathrm{SiH}_{3} \mathrm{~F}$ & 1519.5 & 1525.8 & $1538.5 \pm 20.9^{\mathrm{a}}$ & $1521.6^{\mathrm{j}}, 1525.9^{\mathrm{l}}$ \\
\hline $\mathrm{SiHF}_{2}$ & 1401.8 & 1405.7 & & \\
\hline $\mathrm{SiH}_{2} \mathrm{~F}_{2}$ & 1798.8 & 1806.3 & $1814.9 \pm 20.9^{\mathrm{a}}$ & \\
\hline $\mathrm{SiHF}_{3}$ & 2088.5 & 2096.2 & $2087.5 \pm 20.9^{\mathrm{a}}$ & \\
\hline $\mathrm{SiCl}$ & 422.3 & 421.1 & $369.4 \pm 6.7^{\mathrm{a}}, 425.0^{\mathrm{b}}$ & \\
\hline $\mathrm{SiCl}_{2}$ & 853.7 & 855.6 & $854.0 \pm 3.3^{\mathrm{a}}, 848.1^{\mathrm{b}}$ & \\
\hline $\mathrm{SiCl}_{3}$ & 1125.7 & 1129.2 & $1194.1 \pm 16.7^{\mathrm{a}}$ & \\
\hline $\mathrm{SiCl}_{4}$ & 1583.4 & 1588.6 & $1585.1 \pm 1.3^{\mathrm{a}}, 1584.3^{\mathrm{b}}$ & $1591.1^{\mathrm{f}}$ \\
\hline $\mathrm{SiHCl}$ & 724.9 & 725.6 & & \\
\hline $\mathrm{SiH}_{2} \mathrm{Cl}$ & 963.3 & 967.9 & & $969.0^{1}$ \\
\hline $\mathrm{SiH}_{3} \mathrm{Cl}$ & 1341.2 & 1348.2 & $1346.5 \pm 8^{\mathrm{a}}$ & $1349.2^{1}$ \\
\hline $\mathrm{SiHCl}_{2}$ & 1044.4 & 1049.3 & & \\
\hline $\mathrm{SiH}_{2} \mathrm{Cl}_{2}$ & 1422.0 & 1430.5 & $1430.3 \pm 12.6^{\mathrm{a}}$ & $1431.2^{\mathrm{f}}$ \\
\hline $\mathrm{SiHCl}_{3}$ & 1505.3 & 1513.2 & $1512.0 \pm 4.2^{\mathrm{a}}$ & $1514.9^{f}$ \\
\hline $\mathrm{SiBr}$ & 357.6 & & $323.1 \pm 46^{\mathrm{a}}$ & \\
\hline
\end{tabular}


Table II. (Continued.)

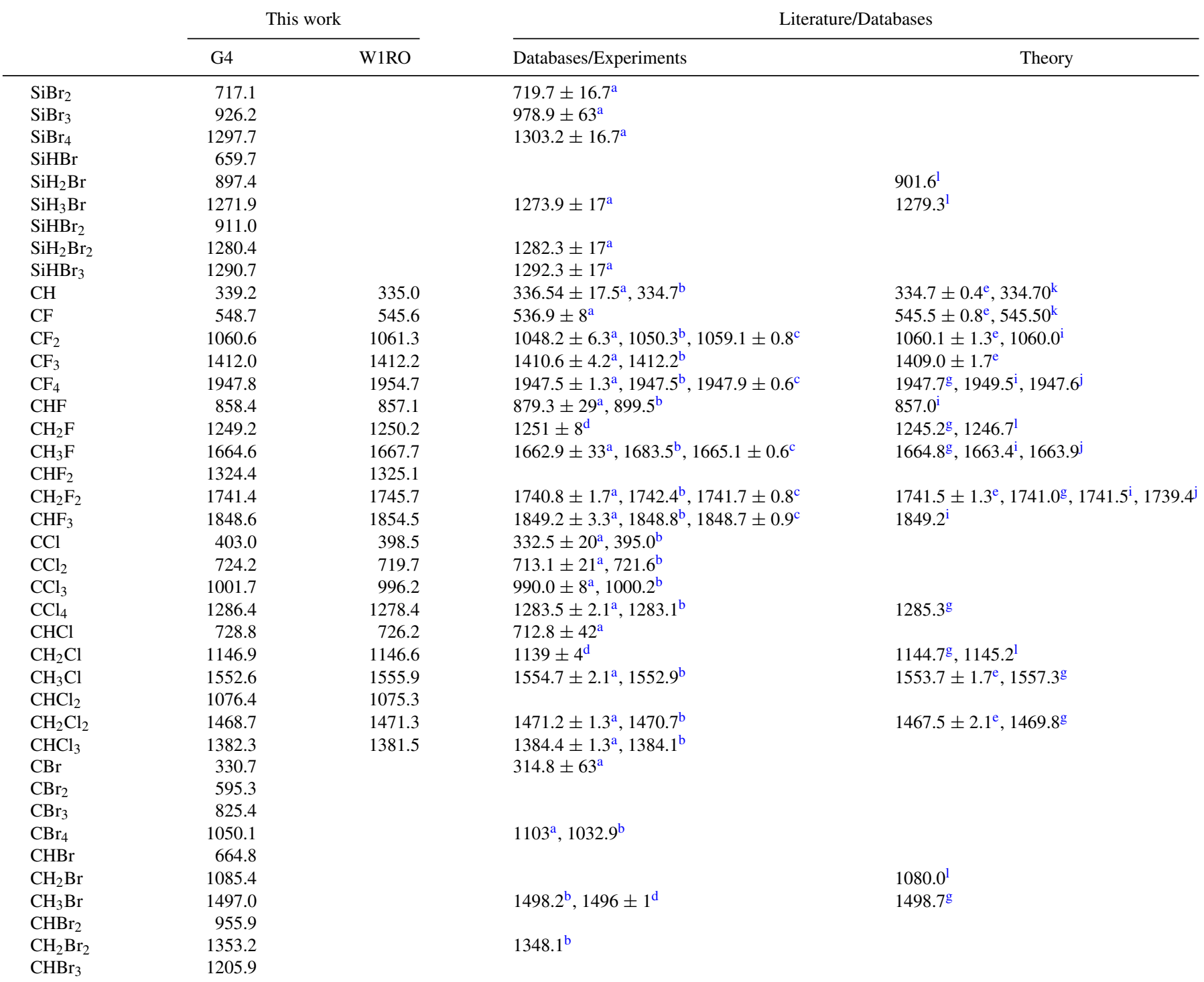

\footnotetext{
${ }^{\text {a Based on }} \Delta \mathrm{H}_{f}^{\circ}(0 \mathrm{~K})$ from NIST-JANAF database, $1998^{6} ;{ }^{\mathrm{b}}$ NIST-CCCBDB database (experiment $)^{40} ;{ }^{\mathrm{c}}$ Active thermochemical table database (ATcT) ${ }^{29}$; ${ }^{\mathrm{d}}$ Feller et al., 2003 (experiment) ${ }^{52}$; ${ }^{\mathrm{e}}$ Feller et al. 2008 (theory) ${ }^{26}$; ${ }^{\mathrm{f}}$ Vasiliiu et al. 2012 (theory) ${ }^{27}$; ${ }^{\mathrm{g}}$ Feller et al. 2003 (theory) ${ }^{52}$; ${ }^{\mathrm{h}}$ Feller and Dixon, 1999 (theory) ${ }^{28 ;}$ i Klopper et al. 2009 (theory) ${ }^{29} ;{ }^{j}$ Karton et al. 2011 (W4 theory) $)^{30}$; ${ }^{\mathrm{k}}$ Tajti et al. (theory) ${ }^{31}$; ${ }^{1}$ Grant and Dixon 2009 (theory). ${ }^{32}$
}

The method however produces small systematic deviations $(\sim 5-10$ $\mathrm{kJ} \mathrm{mol}^{-1}$ ) in the $\mathrm{Si}-\mathrm{Cl}-\mathrm{H}$ compounds, which are possibly caused by slow convergence of the correlation energies despite the increased $d$ polarization sets, as previously observed by Curtiss et al. ${ }^{33}$ It also should be noted that unlike the more expensive calculation procedures such as W1RO, Feller-Peterson-Dixon ${ }^{45}$ etc., G4 do not explicitly include scalar relativistic calculations. It is possible that this omission may not be remedied sufficiently by the HLCs and may affect the accuracy in $\mathrm{Br}$ containing compounds.

Enthalpies of formation $\left(\Delta \mathrm{H}_{f}^{\circ}\right)$ at $O$ and $298 \mathrm{~K}$. - Tables III and IV present $\Delta \mathrm{H}_{f}^{\circ}$ at 0 and $298 \mathrm{~K}$ calculated from the G4 and W1RO $\Sigma \mathrm{D}_{0}$ in comparison to the databases and literature. The G4 and W1RO $\Delta \mathrm{H}_{f}^{\circ}$ utilized the atomic properties respectively from the NIST-CCCBDB, and the NIST-CC-KM - the latter represents the NIST-CCCBDB with its atomic $\mathrm{Si} \Delta \mathrm{H}_{f}^{\circ}(0 \mathrm{~K})$ replaced by the recommended value from Karton and Martin, 2007. ${ }^{46}$ Such matchings are suggested by the lowest deviations comparing to the literature.
The discrepancies observed in the case of diatomic molecules are partly due to the fact that the low-lying electronically excited states of the molecules resulted from spin orbit coupling (SOC) effects need to be taken into account when dealing with the temperature above $0 \mathrm{~K}$. The inclusion of SOC effects are shown within parentheses in Table IV. The low lying excited states were taken into account in the electronic partition function using the following experimental energy splittings (in $\mathrm{cm}^{-1}$ ): 142.830 for $\mathrm{SiH}^{41} ; 161.88$ for $\mathrm{SiF}^{41} ; 207.21$ for $\mathrm{SiCl}^{41}$; 418.0 for $\mathrm{SiBr}^{41}$; 27.950 for $\mathrm{CH}^{41} ; 77.1$ for $\mathrm{CF}^{41}$; 466 for $\mathrm{CBr}^{42}$ and a calculated value of 136.9 for $\mathrm{CCl}^{43}$ The effects increase the enthalpies (in $\mathrm{kJ} \mathrm{mol}^{-1}$ ) by 0.57 for $\mathrm{SiH}$; 0.61 for $\mathrm{SiF} ; 0.67$ for $\mathrm{SiCl}$; 0.59 for $\mathrm{SiBr}$; 0.16 for $\mathrm{CH}$; 0.38 for $\mathrm{CF}$; 0.56 for $\mathrm{CCl}$ and 0.53 for $\mathrm{CBr}$. Despite the small effects for the enthalpies, the inclusion of SOCs significantly improves the agreement between the experimental data and the calculated $\mathrm{S}^{298}$ and $\mathrm{C}_{\mathrm{p}}{ }^{298}$, as shown later in Table V. The experimental and theoretical data of SOC corrections for more complex molecules are however scarce and it is beyond the scope of this study to calculate and include such effects further. The reader is referred to Ref. 44 for details of the SOC calculations. 
Naturally due to their relation to $\Sigma \mathrm{D}_{0}$, we observed similar deviations in $\Delta \mathrm{H}_{f}^{\circ}$ in the set of species discussed previously in the last section, namely the deviation between the databases (NIST-JANAF and NIST-CCCBDB) $\Delta \mathrm{H}_{f}^{\circ}$ and the theoretical results reported in the literature and our calculations. Table IV also includes the calcula- tion results from the ccCA methods ${ }^{47-49}$ for Si-F-H and $\mathrm{Si}-\mathrm{Cl}-\mathrm{H}^{17}$ for further comparison. As reported by DeYonker et al., ${ }^{48}$ the ccCA should give accuracy comparable to the G3X model ${ }^{50}$ and thus is expected to be less accurate than the G4 and W1RO. Nevertheless, good agreement are observed overall between our calculations and

Table III. Enthalpies of formation $\left(\Delta \mathrm{H}_{f}^{\circ}\right)$ at $0 \mathrm{~K}$ in $\mathrm{kJ} \cdot \mathrm{mol}^{-1}$ derived from the G4 (with NIST-CCCBDB atomic properties) and W1RO (with NIST-CC-KM atomic properties) in comparison to literature. Due to the method construction, the W1RO is applied only to the F-and Cl-systems.

This work

Literature

\begin{tabular}{|c|c|c|c|c|}
\hline & & \\
\hline & G4 & W1RO & Databases/Experiments & Theory \\
\hline $\mathrm{SiH}$ & 365.2 & 369.9 & $374.89 \pm 8.4^{\mathrm{a}}, 366.9^{\mathrm{b}}$ & \\
\hline $\mathrm{SiF}$ & -66.8 & -61.9 & $-21.91 \pm 12.6^{\mathrm{a}},-27.06 \pm 11^{\mathrm{b}},-49 \pm 9^{\mathrm{c}}$ & \\
\hline $\mathrm{SiF}_{2}$ & -635.4 & -633.6 & $-587.01 \pm 12.6^{\mathrm{a}},-637 \pm 6^{\mathrm{c}}$ & $-634.7^{\mathrm{j}}$ \\
\hline $\mathrm{SiF}_{3}$ & -997.4 & -997.9 & $-1082.27 \pm 16.7^{\mathrm{a}},-994 \pm 5^{\mathrm{c}}$ & \\
\hline $\mathrm{SiF}_{4}$ & -1609.2 & -1613.3 & $-1609.43 \pm 0.84^{\mathrm{a}},-1609.49 \pm 0.8^{\mathrm{b}}$ & $-1615.9^{j}$ \\
\hline $\mathrm{SiHF}$ & -158.1 & -155.4 & & \\
\hline $\mathrm{SiH}_{2} \mathrm{~F}$ & -176.9 & -177.9 & & $-177.4^{\mathrm{m}}$ \\
\hline $\mathrm{SiH}_{3} \mathrm{~F}$ & -348.5 & -351.9 & $-367.16 \pm 20.9^{\mathrm{a}}$ & $-350.6^{\mathrm{m}}$ \\
\hline $\mathrm{SiHF}_{2}$ & -585.6 & -586.6 & & \\
\hline $\mathrm{SiH}_{2} \mathrm{~F}_{2}$ & -766.5 & -771.2 & $-782.25 \pm 20.9^{\mathrm{a}}$ & \\
\hline $\mathrm{SiHF}_{3}$ & -1195.0 & -1199.8 & $-1193.66 \pm 20.9^{\mathrm{a}}$ & \\
\hline $\mathrm{SiCl}$ & 143.0 & 147.0 & $196.25 \pm 6.7^{\mathrm{a}}, 140.29 \pm 41^{\mathrm{b}}$ & \\
\hline $\mathrm{SiCl}_{2}$ & -168.8 & -167.8 & $-168.74 \pm 3.3^{\mathrm{a}},-163.2 \pm 4.2^{\mathrm{b}}$ & $-168.6^{\mathrm{j}}$ \\
\hline $\mathrm{SiCl}_{3}$ & -321.2 & -321.8 & $-389.25 \pm 16.7^{\mathrm{a}},-320 \pm 8^{\mathrm{c}}$ & \\
\hline $\mathrm{SiCl}_{4}$ & -659.2 & -661.6 & $-660.57 \pm 1.3^{\mathrm{a}},-660.08 \pm 0.80^{\mathrm{b}}$ & $-664.0^{\mathrm{j}},-663.2^{\mathrm{k}}$ \\
\hline $\mathrm{SiHCl}$ & 56.4 & 58.5 & $56.322^{\mathrm{d}}$ & \\
\hline $\mathrm{SiH}_{2} \mathrm{Cl}$ & 34.1 & 32.3 & & $31.8^{\mathrm{m}}$ \\
\hline $\mathrm{SiH}_{3} \mathrm{Cl}$ & -127.7 & -132.0 & $-132.77 \pm 8^{a}$ & $-132.2^{\mathrm{m}}$ \\
\hline $\mathrm{SiHCl}_{2}$ & -143.5 & -145.5 & & \\
\hline $\mathrm{SiH}_{2} \mathrm{Cl}_{2}$ & -305.0 & -310.6 & $-313 \pm 12.6^{\mathrm{a}}$ & $-310.5^{\mathrm{k}}$ \\
\hline $\mathrm{SiHCl}_{3}$ & -484.7 & -489.8 & $-491.15 \pm 4.2^{\mathrm{a}},-489.6 \pm 2.3^{\mathrm{c}}$ & $-490.8^{k}$ \\
\hline $\mathrm{SiBr}$ & 206.0 & & $240.8 \pm 46^{\mathrm{a}}$ & \\
\hline $\mathrm{SiBr}_{2}$ & -35.5 & & $-37.9 \pm 16.7^{\mathrm{a}}$ & \\
\hline $\mathrm{SiBr}_{3}$ & -126.8 & & $-179.1 \pm 63^{\mathrm{a}}$ & \\
\hline $\mathrm{SiBr}_{4}$ & -380.3 & & $-385.55 \pm 16.7^{\mathrm{a}}$ & \\
\hline $\mathrm{SiHBr}$ & 119.9 & & & \\
\hline $\mathrm{SiH}_{2} \mathrm{Br}$ & 98.3 & & & $97.5^{\mathrm{m}}$ \\
\hline $\mathrm{SiH}_{3} \mathrm{Br}$ & -60.2 & & $-61.84 \pm 17^{\mathrm{a}}$ & $-64.0^{\mathrm{m}}$ \\
\hline $\mathrm{SiHBr}_{2}$ & -13.4 & & & \\
\hline $\mathrm{SiH}_{2} \mathrm{Br}_{2}$ & -166.8 & & $-168.4 \pm 17^{\mathrm{a}}$ & \\
\hline $\mathrm{SiHBr}_{3}$ & -275.2 & & $-276.5 \pm 17^{\mathrm{a}}$ & \\
\hline $\mathrm{CH}$ & 588.1 & 592.3 & $590.683 \pm 17.5^{\mathrm{a}}, 592.5^{\mathrm{b}}, 592.96 \pm 0.25^{\mathrm{e}}$ & $593.12^{\mathrm{i}}$ \\
\hline $\mathrm{CF}$ & 239.7 & 242.9 & $251.6 \pm 8^{\mathrm{a}}$ & $243.3 \pm 0.8^{\mathrm{h}}, 243.50^{\mathrm{i}}$ \\
\hline $\mathrm{CF}_{2}$ & -194.9 & -195.5 & $-182.48 \pm 6.3^{\mathrm{a}},-184.6 \pm 8.4^{\mathrm{b}},-191.7 \pm 1.4^{\mathrm{e}}$ & $-193.6 \pm 1.2^{\mathrm{h}},-192.5^{\mathrm{j}}$ \\
\hline $\mathrm{CF}_{3}$ & -469.0 & -469.2 & $-467.52 \pm 4.2^{\mathrm{a}},-469.19 \pm 5^{\mathrm{b}},-462.7 \pm 2^{\mathrm{f}}$ & $-464.8 \pm 1.6^{\mathrm{h}}$ \\
\hline $\mathrm{CF}_{4}$ & -927.5 & -934.4 & $-927.23 \pm 1.3^{\mathrm{a}},-927.23 \pm 0.75^{\mathrm{b}},-927.2 \pm 0.5^{\mathrm{e}}$ & $-927.8 \pm 2.0^{\mathrm{h}},-930.9^{\mathrm{j}}$ \\
\hline $\mathrm{CHF}$ & 146.1 & 147.4 & $125.2 \pm 29^{\mathrm{a}}, 105 \pm 25^{\mathrm{b}}$ & $148.9 \pm 1.2^{\mathrm{h}}$ \\
\hline $\mathrm{CH}_{2} \mathrm{~F}$ & -28.7 & -29.6 & & $-27.9 \pm 1.6^{\mathrm{h}},-26.4^{\mathrm{m}}$ \\
\hline $\mathrm{CH}_{3} \mathrm{~F}$ & -228.1 & -231.1 & $-226.3 \pm 33^{\mathrm{a}},-246.97 \pm 15^{\mathrm{b}},-231.5 \pm 2.7^{\mathrm{e}}$ & $-228.5 \pm 2.0^{\mathrm{h}},-228.1 \pm 1.7^{\mathrm{l}}$ \\
\hline $\mathrm{CHF}_{2}$ & -242.7 & -243.3 & & $-239.4 \pm 2.6^{\mathrm{h}}$ \\
\hline $\mathrm{CH}_{2} \mathrm{~F}_{2}$ & -443.6 & -447.9 & $-443.01 \pm 1.7^{\mathrm{a}},-444.55 \pm 1.7^{\mathrm{b}}$ & $-442.6 \pm 2.0^{\mathrm{h}}$ \\
\hline $\mathrm{CHF}_{3}$ & -689.5 & -695.5 & $-690.1 \pm 3.3^{\mathrm{a}},-689.74 \pm 2.3^{\mathrm{b}},-685.8 \pm 2^{\mathrm{f}}$ & $-687.7 \pm 2.0^{\mathrm{h}},-690.4 \pm 2.1^{\mathrm{l}}$ \\
\hline $\mathrm{CCl}$ & 427.8 & 432.3 & $498.3 \pm 20^{\mathrm{a}}, 435.81 \pm 40^{\mathrm{b}}$ & $430.0 \pm 1.1^{\mathrm{h}}$ \\
\hline $\mathrm{CCl}_{2}$ & 226.2 & 230.7 & $237.3 \pm 21^{\mathrm{a}}, 228.9 \pm 8.4^{\mathrm{b}}$ & $229.0 \pm 1.9^{\mathrm{h}}, 231.0^{\mathrm{j}}$ \\
\hline $\mathrm{CCl}_{3}$ & 68.3 & 73.9 & $80.1 \pm 8^{\mathrm{a}}, 69.9 \pm 2.5^{\mathrm{b}}$ & $73.7 \pm 4.9^{\mathrm{h}}$ \\
\hline $\mathrm{CCl}_{4}$ & -96.7 & -88.8 & $-93.81 \pm 2.1^{\mathrm{a}},-93.35 \pm 1^{\mathrm{b}},-93.20 \pm 0.55^{\mathrm{e}}$ & $-88.7 \pm 6.4^{\mathrm{h}},-89.5^{\mathrm{j}}$ \\
\hline $\mathrm{CHCl}$ & 318.0 & 320.7 & $334 \pm 42^{\mathrm{a}}$ & $320.1 \pm 2.3^{\mathrm{h}}$ \\
\hline $\mathrm{CH}_{2} \mathrm{Cl}$ & 116.0 & 116.3 & & $119.4 \pm 2.7^{\mathrm{h}}, 117.6^{\mathrm{m}}$ \\
\hline $\mathrm{CH}_{3} \mathrm{Cl}$ & -73.6 & -76.9 & $-75.75 \pm 2.1^{\mathrm{a}},-73.94 \pm 0.6^{\mathrm{b}},-74.7 \pm 0.35^{\mathrm{e}}$ & $-74.3 \pm 3.1^{\mathrm{h}}$ \\
\hline $\mathrm{CHCl}_{2}$ & 90.1 & 91.2 & & $91.2 \pm 4.8^{\mathrm{h}}$ \\
\hline $\mathrm{CH}_{2} \mathrm{Cl}_{2}$ & -86.2 & -88.8 & $-88.66 \pm 1.3^{\mathrm{a}},-88.15 \pm 0.3^{\mathrm{b}},-88.55 \pm 0.74^{\mathrm{e}}$ & $-86.5 \pm 4.2^{\mathrm{h}}$ \\
\hline $\mathrm{CHCl}_{3}$ & -96.2 & -95.4 & $-98.27 \pm 1.3^{\mathrm{a}},-97.95 \pm 1.1^{\mathrm{b}},-98.4 \pm 0.8^{\mathrm{e}},-98.4 \pm 1.1^{\mathrm{g}}$ & $-94.6 \pm 5.3^{\mathrm{h}}$ \\
\hline $\mathrm{CBr}$ & 498.4 & & $514.3 \pm 63^{\mathrm{a}}$ & \\
\hline $\mathrm{CBr}_{2}$ & 351.8 & & $356.89^{\mathrm{a}}$ & \\
\hline $\mathrm{CBr}_{3}$ & 239.6 & & & \\
\hline $\mathrm{CBr}_{4}$ & 132.8 & & $79.89^{\mathrm{a}}, 150 \pm 15^{\mathrm{b}}$ & \\
\hline
\end{tabular}


Table III. (Continued.)

\begin{tabular}{|c|c|c|c|c|}
\hline & \multicolumn{2}{|c|}{ This work } & \multicolumn{2}{|c|}{ Literature } \\
\hline $\mathrm{CHBr}$ & 380.4 & & & \\
\hline $\mathrm{CH}_{3} \mathrm{Br}$ & -19.8 & & $-21 \pm 0.5^{\mathrm{b}}$ & \\
\hline $\mathrm{CHBr}_{2}$ & 207.1 & & & \\
\hline $\mathrm{CH}_{2} \mathrm{Br}_{2}$ & 26.0 & & $31 \pm 15^{\mathrm{b}}$ & \\
\hline
\end{tabular}

${ }^{a}$ NIST-JANAF database ${ }^{6} ;{ }^{b}$ NIST-CCCBDB database (experiments) ${ }^{40}$; ${ }^{\mathrm{c}}$ Shuman et al., 2009 (experiments) ${ }^{13}$; ${ }^{\mathrm{d}}$ Deng et al., 2008 (experiments) ${ }^{53}$; ${ }^{\mathrm{e}}$ ATcT database ${ }^{20}$; ${ }^{\mathrm{f}}$ Ruscic et al., 1998 (evaluation) ${ }^{54}$; ${ }^{\mathrm{g}}$ Shuman et al., 2008 (experiments) ${ }^{12}$; ${ }^{\mathrm{h}}$ Csontos et al. 2010 (theory) ${ }^{20}$; i Tajti et al. 2004 (theory) ${ }^{31}$; ${ }^{\text {j}}$ Thanthiriwatte et al. 2015 (theory) ${ }^{55}$; ${ }^{\mathrm{k}}$ Vasiliu et al. 2012 (theory) ${ }^{27}$; ${ }^{\mathrm{l}}$ Feller et al. 2012 (theory) ${ }^{56}$; ${ }^{\mathrm{m}}$ Grant and Dixon 2009 (theory). ${ }^{32}$

Table IV. Enthalpies of formation $\left(\Delta \mathrm{H}_{f}^{\circ}\right)$ at $298 \mathrm{~K}$ in $\mathrm{kJ} \cdot \mathrm{mol}^{-1}$ derived from the G4 (with NIST-CCCBDB atomic properties) and W1RO (with NIST-CC-KM atomic properties) in comparison to literature. Due to the method construction, the W1RO is applied only to the F-and Cl-systems. The parentheses present the result when the effects of low lying excited states due to spin orbit coupling (SOC) are included.

This work

\begin{tabular}{|c|c|c|c|c|}
\hline & G4 & W1RO & Databases/Experiments & Theory \\
\hline $\mathrm{SiH}$ & $\begin{array}{c}366.4 \\
(366.9)\end{array}$ & $\begin{array}{c}371.2 \\
(371.7)\end{array}$ & $376.66 \pm 8.4^{\mathrm{a}}, 368.64^{\mathrm{b}}, 361.0^{\mathrm{d}}$ & $371.9^{\text {aa }}$ \\
\hline $\mathrm{SiF}$ & $\begin{array}{l}-65.6 \\
(-65.0)\end{array}$ & $\begin{array}{l}-60.7 \\
(-60.1)\end{array}$ & $-20.083 \pm 12.6^{\mathrm{a}},-25.23 \pm 11^{\mathrm{b}},-47 \pm 9^{\mathrm{c}}, 7.1^{\mathrm{d}},-21 \pm 25^{\mathrm{e}}$ & $-58.2^{\text {aa }}$ \\
\hline $\mathrm{SiF}_{2}$ & -636.2 & -634.4 & $-587.852 \pm 12.6^{\mathrm{a}},-638 \pm 6^{\mathrm{c}},-619.0^{\mathrm{d}},-590 \pm 8^{\mathrm{e}},-595.8 \pm 7^{\mathrm{f}}$ & $-635.5^{\mathrm{y}},-631.1^{\mathrm{aa}}$ \\
\hline $\mathrm{SiF}_{3}$ & -1000.4 & -1000.8 & $-1085.33 \pm 16.7^{\mathrm{a}},-998 \pm 4.6^{\mathrm{c}},-1000 \pm 21^{\mathrm{e}},-1075 \pm 11^{\mathrm{f}}$ & $-988.1^{\text {aa }}$ \\
\hline $\mathrm{SiF}_{4}$ & -1614.6 & -1618.6 & $-1614.94 \pm 0.84^{\mathrm{a}},-1615.0 \pm 0.8^{\mathrm{b}},-1615.78 \pm 0.5^{\mathrm{g}}$ & $-1621.3^{\mathrm{y}},-1610.4^{\mathrm{z}},-1605.9^{\mathrm{aa}}$ \\
\hline $\mathrm{SiHF}$ & -159.7 & -157.0 & & $-156.2^{\text {aa }}$ \\
\hline $\mathrm{SiH}_{2} \mathrm{~F}$ & -182.3 & -183.2 & & $-177.1^{\text {aa }}$ \\
\hline $\mathrm{SiH}_{3} \mathrm{~F}$ & -357.8 & -361.3 & $-376.56 \pm 20.9^{\mathrm{a}},-415.9 \pm 8^{\mathrm{h}}$ & $-357.2^{\mathrm{aa}}$ \\
\hline $\mathrm{SiHF}_{2}$ & -590.1 & -591.0 & & $-582.0^{\mathrm{aa}}$ \\
\hline $\mathrm{SiH}_{2} \mathrm{~F}_{2}$ & -775.0 & -779.6 & $-790.776 \pm 20.9^{\mathrm{a}},-804.2 \pm 8^{\mathrm{h}}$ & $-772.9^{\text {aa }}$ \\
\hline $\mathrm{SiHF}_{3}$ & -1202.0 & -1206.8 & $-1200.808 \pm 20.9^{\mathrm{a}},-1225.9 \pm 8^{\mathrm{h}}$ & $-1197.3^{\text {aa }}$ \\
\hline $\mathrm{SiCl}$ & $\begin{array}{c}144.5 \\
(145.2)\end{array}$ & $\begin{array}{c}148.5 \\
(149.2)\end{array}$ & $198.32 \pm 6.7^{\mathrm{a}}, 142.36 \pm 41^{\mathrm{b}}, 189.9^{\mathrm{d}}, 155 \pm 42^{\mathrm{e}}, 154.8 \pm 4^{\mathrm{i}}$ & $153.0^{\text {aa }}$ \\
\hline $\mathrm{SiCl}_{2}$ & -168.5 & -167.6 & $-168.62 \pm 3.3^{\mathrm{a}, \mathrm{e}},-163.07 \pm 4.2^{\mathrm{b}},-151 \pm 8^{\mathrm{i}},-159 \pm 6^{\mathrm{i}}$ & $-168.2^{\mathrm{y}},-161.3^{\mathrm{aa}}$ \\
\hline $\mathrm{SiCl}_{3}$ & -321.9 & -322.6 & $-390.37 \pm 16.7^{\mathrm{a}},-334.7 \pm 8^{\mathrm{e}},-318 \pm 4^{\mathrm{i}},-321 \pm 8^{\mathrm{j}},-326 \pm 12^{\mathrm{j}}$ & $-307.3^{\text {aa }}$ \\
\hline $\mathrm{SiCl}_{4}$ & -661.0 & -663.4 & $-662.75 \pm 1.3^{\mathrm{a}},-662.2 \pm 0.8^{\mathrm{b}},-657.0^{\mathrm{d}}$ & $-666.1^{\mathrm{y}},-665.3^{\mathrm{z}}, 648.0^{\mathrm{aa}}$ \\
\hline $\mathrm{SiHCl}$ & 55.1 & 57.2 & $54.945^{\mathrm{k}}$ & $59.9^{\text {aa }}$ \\
\hline $\mathrm{SiH}_{2} \mathrm{Cl}$ & 29.1 & 27.2 & & $34.9^{\text {aa }}$ \\
\hline $\mathrm{SiH}_{3} \mathrm{Cl}$ & -136.7 & -140.9 & $-141.84 \pm 8^{\mathrm{a}},-135.6 \pm 10.5^{\mathrm{e}}$ & $-141.4^{\mathrm{z}},-135.5^{\mathrm{aa}}$ \\
\hline $\mathrm{SiHCl}_{2}$ & -146.9 & -148.9 & & $-137.3^{\text {aa }}$ \\
\hline $\mathrm{SiH}_{2} \mathrm{Cl}_{2}$ & -312.3 & -317.9 & $-320.49^{\mathrm{a}},-315.1 \pm 8.4^{\mathrm{e}}$ & $-318.0^{\mathrm{z}},-308.9^{\mathrm{aa}}$ \\
\hline $\mathrm{SiHCl}_{3}$ & -489.5 & -494.6 & $-496.22 \pm 4.2^{\mathrm{a}},-513.00^{\mathrm{d}},-494.4 \pm 2.3^{\mathrm{j}},-499.2 \pm 7^{\mathrm{j}}$ & $-495.8^{\mathrm{z}},-482.3^{\mathrm{aa}}$ \\
\hline $\mathrm{SiBr}$ & $\begin{array}{c}200.0 \\
(200.6)\end{array}$ & & $235.3 \pm 46^{\mathrm{a}}, 209.0^{\mathrm{d}}, 197 \pm 42^{\mathrm{e}}, 204 \pm 8^{\mathrm{i}}$ & \\
\hline $\mathrm{SiBr}_{2}$ & -49.8 & & $-52.3 \pm 16.7^{\mathrm{a}},-46 \pm 8.4^{\mathrm{e}},-46 \pm 4^{\mathrm{i}}$ & \\
\hline $\mathrm{SiBr}_{3}$ & -148.8 & & $-201.7 \pm 63^{\mathrm{a}},-159 \pm 8^{\mathrm{i}},-159 \pm 25^{\mathrm{e}}$ & \\
\hline $\mathrm{SiBr}_{4}$ & -410.0 & & $-415.47 \pm 16.7^{\mathrm{a}},-415.5^{\mathrm{d}},-416 \pm 5^{\mathrm{i}},-415.5 \pm 8^{\mathrm{e}}$ & \\
\hline $\mathrm{SiHBr}$ & 111.2 & & $-464.4^{\mathrm{d}}$ & \\
\hline $\mathrm{SiH}_{2} \mathrm{Br}$ & 85.9 & & & \\
\hline $\mathrm{SiH}_{3} \mathrm{Br}$ & -76.5 & & $-78.24 \pm 17^{\mathrm{a}},-64.0 \pm 9^{\mathrm{e}}$ & \\
\hline $\mathrm{SiHBr}_{2}$ & -31.3 & & & \\
\hline $\mathrm{SiH}_{2} \mathrm{Br}_{2}$ & -188.6 & & $-190.4 \pm 17^{\mathrm{a}},-180.8 \pm 10^{\mathrm{e}}$ & \\
\hline $\mathrm{SiHBr}_{3}$ & -301.3 & & $-302.9 \pm 17^{\mathrm{a}},-317.6^{\mathrm{d}},-303.3 \pm 9^{\mathrm{e}}$ & \\
\hline $\mathrm{CH}$ & $\begin{array}{c}591.4 \\
(591.6)\end{array}$ & $\begin{array}{c}595.7 \\
(595.8)\end{array}$ & $594.128 \pm 17.5^{\mathrm{a}}, 594.13^{\mathrm{b}}$ & $596.2 \pm 0.4^{\mathrm{ad}}$ \\
\hline $\mathrm{CF}$ & $\begin{array}{l}243.0 \\
(243.4)\end{array}$ & $\begin{array}{l}246.1 \\
(246.5)\end{array}$ & $255.2 \pm 8^{\mathrm{a}}, 246.9 \pm 0.7^{\mathrm{l}}$ & $247.0 \pm 0.8^{\mathrm{x}}, 246.4 \pm 0.8^{\mathrm{ad}}$ \\
\hline $\mathrm{CF}_{2}$ & -194.4 & -195.0 & $\begin{array}{l}-182.0 \pm 6.3^{\mathrm{a}},-184.1 \pm 8.4^{\mathrm{b}},-172 \pm 8.4^{\mathrm{m}},-191.3 \pm 1.4^{\mathrm{l}}, \\
-205 \pm 13^{\mathrm{n}},-171.1 \pm 10^{\mathrm{n}}\end{array}$ & $-193.2 \pm 1.2^{\mathrm{x}},-192.0^{\mathrm{y}},-194.1 \pm 1.3^{\mathrm{ad}}$ \\
\hline $\mathrm{CF}_{3}$ & -471.7 & -471.9 & $\begin{array}{l}-470.28 \pm 4.2^{\mathrm{a}},-472 \pm 5^{\mathrm{b}},-477.0^{\mathrm{d}},-429 . \pm 13^{\mathrm{m}},-465.7 \pm 2^{\mathrm{o}} \\
-467.4 \pm 2.0^{\mathrm{l}}\end{array}$ & $-467.6 \pm 1.6^{\mathrm{x}},-469.0 \pm 1.7^{\mathrm{ad}}$ \\
\hline $\mathrm{CF}_{4}$ & -933.4 & -940.2 & $-933.2 \pm 1.3^{\mathrm{a}},-933.2 \pm 0.75^{\mathrm{b}},-933.6^{\mathrm{d}},-930 \pm 20^{\mathrm{m}},-933.4 \pm 0.5^{\mathrm{l}}$ & $-933.8 \pm 2.0^{\mathrm{x}},-936.8^{\mathrm{y}},-933.0^{\mathrm{ac}}$ \\
\hline $\mathrm{CHF}$ & 146.4 & 147.7 & $125.2 \pm 29^{\mathrm{a}}, 105.29 \pm 25^{\mathrm{b}}, 109 \pm 13^{\mathrm{n}}, 156.9 \pm 18^{\mathrm{n}}, 143.1 \pm 13^{\mathrm{n}}$ & $149.0 \pm 1.2^{\mathrm{x}}$ \\
\hline
\end{tabular}


Table IV. (Continued.)

This work

\begin{tabular}{|c|c|c|c|c|}
\hline & G4 & W1RO & Databases/Experiments & Theory \\
\hline $\mathrm{CH}_{2} \mathrm{~F}$ & -32.1 & -32.9 & $-32 \pm 8.4^{\mathrm{p}},-34 \pm 8^{\mathrm{q}}$ & $-31.2 \pm 1.6^{\mathrm{x}},-28.0^{\mathrm{ac}}$ \\
\hline $\mathrm{CH}_{3} \mathrm{~F}$ & -236.1 & -239.1 & $-236.577 \pm 0.3^{\mathrm{a}},-255 \pm 15^{\mathrm{b}},-247^{\mathrm{m}},-239.6 \pm 2.7^{1},-234 \pm 4^{\mathrm{q}}$ & $-236.9 \pm 2.0^{\mathrm{x}},-236.4^{\mathrm{ac}},-236.1 \pm 1.7^{\mathrm{ab}, \mathrm{ad}}$ \\
\hline $\mathrm{CHF}_{2}$ & -246.1 & -246.7 & $-239 \pm 4.2^{\mathrm{p}}$ & $-243.0 \pm 3.6^{\mathrm{x}}$ \\
\hline $\mathrm{CH}_{2} \mathrm{~F}_{2}$ & -451.3 & -455.5 & $-450.659 \pm 1.7^{\mathrm{a}},-452.2 \pm 1.7^{\mathrm{b}},-452.3^{\mathrm{d}},-452.21 \pm 0.92^{\mathrm{m}}$ & $-450.5 \pm 2.0^{\mathrm{x}},-450.6^{\mathrm{ac}}$ \\
\hline $\mathrm{CHF}_{3}$ & -696.5 & -702.3 & $\begin{array}{l}-697.05 \pm 3.3^{\mathrm{a}},-696.7 \pm 2.3^{\mathrm{b}},-690.8^{\mathrm{m}},-695.4 \pm 2.7^{\mathrm{m}} \\
-695.3 \pm 2.0^{\mathrm{l}},-692.9 \pm 2^{\mathrm{o}}\end{array}$ & $-694.9 \pm 2.0^{\mathrm{x}},-697.3 \pm 2.1^{\mathrm{ab}, \mathrm{ad}}$ \\
\hline $\mathrm{CCl}$ & $\begin{array}{l}431.0 \\
(431.6)\end{array}$ & $\begin{array}{c}435.5 \\
(436.1)\end{array}$ & $502.1 \pm 20^{\mathrm{a}}, 439.57 \pm 40^{\mathrm{b}}$ & $433.7 \pm 1.1^{\mathrm{x}}$ \\
\hline $\mathrm{CCl}_{2}$ & 227.5 & 232.0 & $\begin{array}{l}238.5 \pm 21^{\mathrm{a}}, 221.8 \pm 11^{\mathrm{r}}, 230.1 \pm 8^{\mathrm{n}}, 163 \pm 13^{\mathrm{n}}, 239.3 \pm 17^{\mathrm{n}} \\
213.4 \pm 8^{\mathrm{n}}, 218.0 \pm 14^{\mathrm{n}}\end{array}$ & $230.1 \pm 1.9^{\mathrm{x}}, 232.2^{\mathrm{y}}$ \\
\hline $\mathrm{CCl}_{3}$ & 67.9 & 73.4 & $79.5 \pm 8^{\mathrm{a}}, 71.1 \pm 2.5^{\mathrm{b}}, 59.0^{\mathrm{d}}, 75.3 \pm 8^{\mathrm{s}}$ & $73.1 \pm 4.9^{\mathrm{x}}$ \\
\hline $\mathrm{CCl}_{4}$ & -98.6 & -90.7 & $\begin{array}{l}-95.98 \pm 2.1^{\mathrm{a}},-95.6 \pm 1^{\mathrm{b}},-95.6 \pm 2.5^{\mathrm{m}},-94 \pm 2^{\mathrm{m}},-115 \pm 3^{\mathrm{m}} \\
-125 \pm 4.6^{\mathrm{m}},-103 \pm 7.9^{\mathrm{m}}\end{array}$ & $-91.0 \pm 6.4^{\mathrm{x}},-92.0^{\mathrm{y}},-93.3^{\mathrm{ac}}$ \\
\hline $\mathrm{CHCl}$ & 318.3 & 321.0 & $\begin{array}{l}335 \pm 42^{\mathrm{a}}, 312.1 \pm 10^{\mathrm{r}}, 326.4 \pm 8^{\mathrm{n}}, 297 \pm 21^{\mathrm{n}}, 316.7 \pm 20.1^{\mathrm{n}} \\
336.4 \pm 11^{\mathrm{n}}\end{array}$ & $320.3 \pm 2.3^{x}$ \\
\hline $\mathrm{CH}_{2} \mathrm{Cl}$ & 114.2 & 113.8 & $121 \pm 4^{\mathrm{q}}, 117.3 \pm 3.1^{\mathrm{t}}, 115.9 \pm 8^{\mathrm{s}}$ & $116.0 \pm 2.7^{\mathrm{x}}, 115.5^{\mathrm{ac}}$ \\
\hline $\mathrm{CH}_{3} \mathrm{Cl}$ & -81.5 & -84.8 & $\begin{array}{l}-83.68 \pm 2.1^{\mathrm{a}},-81.9 \pm 1.5^{\mathrm{m}},-81.96 \pm 0.67^{\mathrm{m}},-85.90 \pm 0.59^{\mathrm{m}} \\
-82.6 \pm 0.4^{1}\end{array}$ & $-82.6 \pm 3.1^{\mathrm{x}},-86.2^{\mathrm{ac}}$ \\
\hline $\mathrm{CHCl}_{2}$ & 87.8 & 89.0 & $80 \pm 12^{\mathrm{u}}, 89.0 \pm 3.0^{\mathrm{t}}, 93.3 \pm 8^{\mathrm{s}}$ & $88.8 \pm 5.8^{\mathrm{x}}$ \\
\hline $\mathrm{CH}_{2} \mathrm{Cl}_{2}$ & -93.0 & -95.6 & $\begin{array}{l}-95.521 \pm 1.3^{\mathrm{a}},-95 \pm 0.3^{\mathrm{b}},-95.1 \pm 2.5^{\mathrm{m}},-95.7 \pm 1.3^{\mathrm{m}} \\
-95.4 \pm 0.7^{1}\end{array}$ & $-93.7 \pm 4.2^{\mathrm{x}},-93.7^{\mathrm{ac}}$ \\
\hline $\begin{array}{l}\mathrm{CHCl}_{3} \\
\mathrm{CBr}\end{array}$ & $\begin{array}{c}-100.9 \\
494.0 \\
(494.6)\end{array}$ & -100.1 & $\begin{array}{l}-103.18 \pm 1.3^{\mathrm{a}},-102.7 \pm 1.1^{\mathrm{b}},-102.9 \pm 2.5^{\mathrm{m}},-103.3 \pm 0.8^{\mathrm{l}} \\
510.4 \pm 63^{\mathrm{a}}\end{array}$ & $-99.7 \pm 5.3^{\mathrm{x}}$ \\
\hline $\mathrm{CBr}_{2}$ & 338.5 & & & \\
\hline $\mathrm{CBr}_{3}$ & 217.9 & & $235.0 \pm 25^{\mathrm{w}}, 205 \pm 8^{\mathrm{s}}$ & \\
\hline $\mathrm{CBr}_{4}$ & 103.3 & & $50.208^{\mathrm{a}}, 120 \pm 15^{\mathrm{b}}, 83.9 \pm 3.4^{\mathrm{m}}$ & \\
\hline $\mathrm{CHBr}$ & 373.2 & & & \\
\hline $\mathrm{CH}_{2} \mathrm{Br}$ & 165.9 & & $173.6^{\mathrm{w}}, 169.0 \pm 4^{\mathrm{w}}, 168.2 \pm 8^{\mathrm{s}}$ & \\
\hline $\mathrm{CH}_{3} \mathrm{Br}$ & -35.2 & & $-36.4 \pm 0.5^{\mathrm{b}},-35.4^{\mathrm{d}},-38 \pm 1.3^{\mathrm{m}},-34.3 \pm 0.8^{\mathrm{m}},-37.5 \pm 1.5^{\mathrm{m}}$ & $-36.8^{\mathrm{ac}}$ \\
\hline $\mathrm{CHBr}_{2}$ & 190.3 & & $182 \pm 20^{\mathrm{u}}, 188.3 \pm 8^{\mathrm{w}}, 185.8 \pm 8^{\mathrm{s}}, 227.2^{\mathrm{w}}$ & \\
\hline $\mathrm{CH}_{2} \mathrm{Br}_{2}$ & 4.6 & & $10 \pm 15^{\mathrm{b}},-11.1 \pm 5^{\mathrm{w}}$ & \\
\hline $\mathrm{CHBr}_{3}$ & 49.2 & & $55.4 \pm 3.30^{\mathrm{b}}, 23.8^{\mathrm{d}}, 55.1 \pm 4.9^{\mathrm{v}}, 60.0 \pm 15^{\mathrm{w}}$ & \\
\hline
\end{tabular}

${ }^{\mathrm{a}}$ NIST-JANAF database ${ }^{6} ;{ }^{\mathrm{b}}$ NIST-CCCBDB database (experiments) ${ }^{40}$; ${ }^{\mathrm{c}}$ Fisher et al., 1993 (experiments) ${ }^{15}$; ${ }^{\mathrm{d}}$ CRC database ${ }^{8}$; ${ }^{\mathrm{e}}$ Walsh, 1983 (evaluations) ${ }^{9}$; ${ }^{\mathrm{f}}$ Weber and Armentrout, 1988 (experiments) ${ }^{57}$; g Martin and Taylor, 1999 (experiments) ${ }^{58}$; ${ }^{\text {h}}$ Farber and Srivastava, 1977 (experiments) ${ }^{59}$; i Hildenbrand

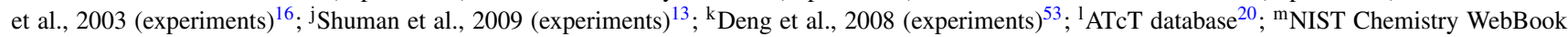
database (experiments) ${ }^{60}$; ${ }^{\mathrm{n}}$ Poutsma et al., 1997 (experiments) ${ }^{10}$; ${ }^{\circ}$ Ruscic et al., 1998 (evaluation) ${ }^{54}$; ${ }^{\mathrm{p}}$ Schwartz et al., 2003 (experiments) ${ }^{24}$; ${ }^{\mathrm{q}} \mathrm{Feller}$ et al., 2003 (experiments) ${ }^{52}$; ${ }^{\mathrm{r}}$ Tian and Kass, 2007 (experiments) ${ }^{14}$; ${ }^{\mathrm{s}}$ Holmes and Lossing, 1988 (experiments) ${ }^{61}$; ${ }^{\mathrm{t}}$ Seetula 1996 (experiments) ${ }^{62}$; ${ }^{\mathrm{u} B o r n}$ et al., 2000 (experiments) ${ }^{11}$; ${ }^{\mathrm{v}}$ Shuman et al., 2008 (experiments) ${ }^{12}$; ${ }^{\mathrm{w}}$ Paddison and Tschuikow-Roux, 1998 (experiments) ${ }^{23}$; ${ }^{\mathrm{x}}$ Csontos et al. 2010 (theory) ${ }^{20}$; ${ }^{\mathrm{y}}$ Thanthiriwatte et al. 2015 (theory) ${ }^{55}$; ${ }^{\mathrm{z}}$ Vasiliu et al. 2012 (theory) ${ }^{27}$; aa Prascher et al. 2009 (theory) ${ }^{17}$; ab Feller et al. 2012 (theory) ${ }^{56}$; ac Feller et al. 2003 (theory) ${ }^{52}$; ad Feller et al. 2008 (theory). ${ }^{26}$

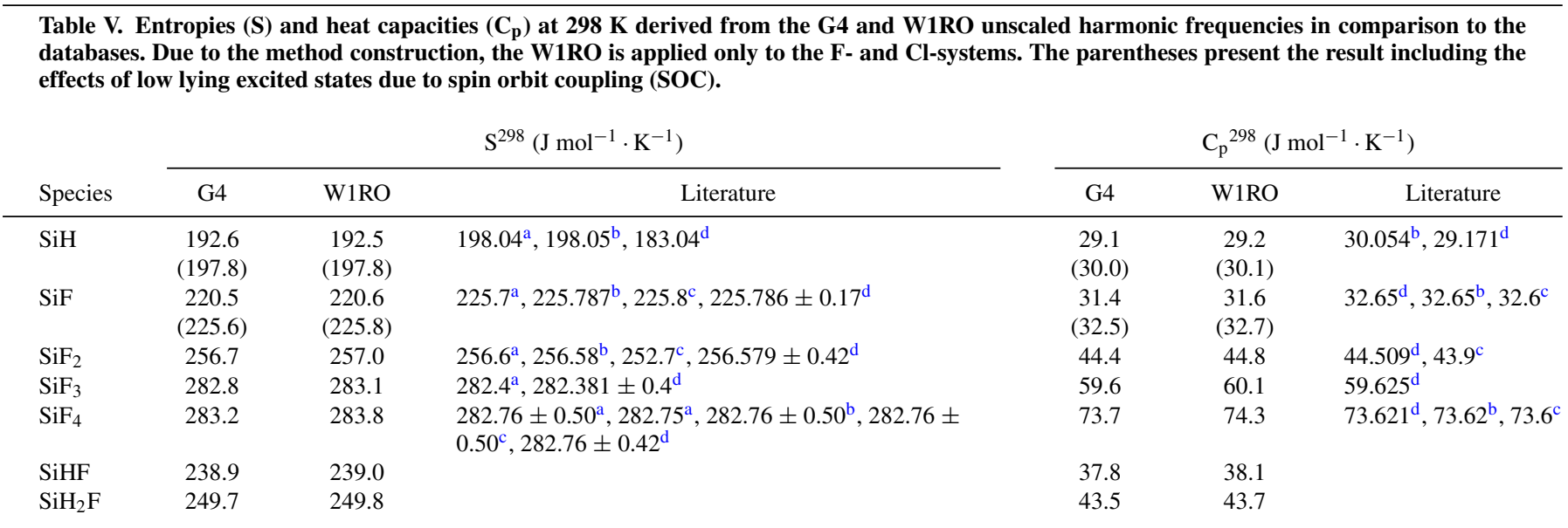


Table V. (Continued.)

\begin{tabular}{|c|c|c|c|c|c|c|}
\hline \multirow[b]{2}{*}{ Species } & \multicolumn{3}{|r|}{$\mathrm{S}^{298}\left(\mathrm{~J} \mathrm{~mol}^{-1} \cdot \mathrm{K}^{-1}\right)$} & \multicolumn{3}{|c|}{$\mathrm{C}_{\mathrm{p}}{ }^{298}\left(\mathrm{~J} \mathrm{~mol}^{-1} \cdot \mathrm{K}^{-1}\right)$} \\
\hline & G4 & W1RO & Literature & G4 & W1RO & Literature \\
\hline $\mathrm{SiH}_{3} \mathrm{~F}$ & 238.2 & 238.3 & $238.4^{\mathrm{a}}, 238.402 \pm 0.80^{\mathrm{b}}, 238.4^{\mathrm{c}}, 238.402 \pm 0.8^{\mathrm{d}}$ & 47.0 & 47.2 & $47.196^{\mathrm{d}}, 47.17^{\mathrm{b}}, 47.4^{\mathrm{c}}$ \\
\hline $\mathrm{SiHF}_{2}$ & 271.9 & 272.1 & & 50.5 & 50.8 & \\
\hline $\mathrm{SiH}_{2} \mathrm{~F}_{2}$ & 262.2 & 262.4 & $262.1^{\mathrm{a}}, 262.130 \pm 2.1^{\mathrm{d}}$ & 54.2 & 54.5 & $54.268^{\mathrm{d}}$ \\
\hline $\mathrm{SiHF}_{3}$ & 277.7 & 278.0 & $277.3^{\mathrm{a}}, 271.9^{\mathrm{c}}, 277.268 \pm 0.8^{\mathrm{d}}$ & 63.6 & 64.0 & $63.485^{\mathrm{d}}, 60.5^{\mathrm{c}}$ \\
\hline $\mathrm{SiCl}$ & $\begin{array}{c}233.0 \\
(237.9)\end{array}$ & $\begin{array}{c}233.0 \\
(237.8)\end{array}$ & $237.81^{\mathrm{a}}, 237.837 \pm 0.21^{\mathrm{b}}, 237.833 \pm 0.21^{\mathrm{d}}$ & $\begin{array}{c}34.2 \\
(35.8)\end{array}$ & $\begin{array}{c}34.2 \\
(35.8)\end{array}$ & $35.78^{\mathrm{d}}, 35.78^{\mathrm{b}}, 36.9^{\mathrm{c}}$ \\
\hline $\mathrm{SiCl}_{2}$ & 282.4 & 282.4 & $281.3^{\mathrm{a}}, 281.613^{\mathrm{b}}, 281.333 \pm 0.8^{\mathrm{d}}$ & 51.5 & 51.5 & $51.248^{\mathrm{d}}, 51.27^{\mathrm{b}}$ \\
\hline $\mathrm{SiCl}_{3}$ & 321.0 & 320.9 & $318.13^{\mathrm{a}}, 318.13^{\mathrm{b}}, 318.189 \pm 4.2^{\mathrm{d}}$ & 71.5 & 71.5 & $70.733^{\mathrm{d}}$ \\
\hline $\mathrm{SiCl}_{4}$ & 332.6 & 332.3 & $330.86^{\mathrm{a}}, 331.446^{\mathrm{b}}, 330.7^{\mathrm{c}}, 330.945 \pm 0.21^{\mathrm{d}}$ & 91.0 & 91.0 & $90.261^{\mathrm{d}}, 90.4^{\mathrm{b}}, 90.3^{\mathrm{c}}$ \\
\hline $\mathrm{SiHCl}$ & 251.5 & 251.5 & & 41.1 & 41.2 & \\
\hline $\mathrm{SiH}_{2} \mathrm{Cl}$ & 262.3 & 262.2 & & 47.4 & 47.4 & \\
\hline $\mathrm{SiH}_{3} \mathrm{Cl}$ & 250.8 & 250.7 & $250.76^{\mathrm{a}}, 250.761 \pm 0.21^{\mathrm{b}}, 250.7^{\mathrm{c}}, 250.761 \pm 0.21^{\mathrm{d}}$ & 51.2 & 51.2 & $51.098^{\mathrm{d}}, 51.08^{\mathrm{b}}, 51^{\mathrm{c}}$ \\
\hline $\mathrm{SiHCl}_{2}$ & 297.1 & 297.0 & & 58.0 & 58.1 & \\
\hline $\mathrm{SiH}_{2} \mathrm{Cl}_{2}$ & 287.2 & 287.1 & $286.72^{\mathrm{a}}, 286.734 \pm 0.34^{\mathrm{b}}, 285.7^{\mathrm{c}}, 286.734 \pm 0.34^{\mathrm{d}}$ & 62.5 & 62.5 & $62.174^{\mathrm{d}}, 62.17^{\mathrm{b}}, 60.5^{\mathrm{c}}$ \\
\hline $\mathrm{SiHCl}_{3}$ & 314.9 & 314.7 & $313.71^{\mathrm{a}}, 313.717 \pm 0.40^{\mathrm{b}}, 313.9^{\mathrm{c}}, 313.717 \pm 0.4^{\mathrm{d}}$ & 76.0 & 76.1 & $75.455^{\mathrm{d}}, 75.44^{\mathrm{b}}, 75.8^{\mathrm{c}}$ \\
\hline $\mathrm{SiBr}$ & $\begin{array}{c}244.6 \\
(247.6)\end{array}$ & - & $247.51^{\mathrm{a}}, 247.42 \pm 0.21^{\mathrm{d}}$ & $\begin{array}{c}35.2 \\
(38.7)\end{array}$ & - & $38.731^{\mathrm{d}}, 38.6^{\mathrm{c}}$ \\
\hline $\mathrm{SiBr}_{2}$ & 306.2 & - & $305.19^{\mathrm{a}}, 305.22 \pm 2.13^{\mathrm{d}}$ & 53.8 & - & $53.637^{\mathrm{d}}$ \\
\hline $\mathrm{SiBr}_{3}$ & 357.7 & - & $351.75^{\mathrm{a}}, 351.77 \pm 8.4^{\mathrm{d}}$ & 75.8 & - & $74.578^{\mathrm{d}}$ \\
\hline $\mathrm{SiBr}_{4}$ & 381.2 & - & $379.33^{\mathrm{a}}, 377.9^{\mathrm{c}}, 379.36 \pm 0.8^{\mathrm{d}}$ & 97.5 & - & $97.009^{\mathrm{d}}, 97.1^{\mathrm{c}}$ \\
\hline $\mathrm{SiHBr}$ & 263.2 & - & & 42.4 & - & \\
\hline $\mathrm{SiH}_{2} \mathrm{Br}$ & 274.1 & - & & 49.0 & - & \\
\hline $\mathrm{SiH}_{3} \mathrm{Br}$ & 262.6 & - & $262.46^{\mathrm{a}}, 262.4^{\mathrm{c}}, 262.47 \pm 0.21^{\mathrm{d}}$ & 53.0 & - & $52.865^{\mathrm{d}}, 52.8^{\mathrm{c}}$ \\
\hline $\mathrm{SiHBr}_{2}$ & 321.1 & - & & 61.1 & - & \\
\hline $\mathrm{SiH}_{2} \mathrm{Br}_{2}$ & 311.0 & - & $310.0^{\mathrm{a}}, 309.7^{\mathrm{c}}, 310.05 \pm 1.3^{\mathrm{d}}$ & 65.9 & - & $65.545^{\mathrm{d}}, 65.5^{\mathrm{c}}$ \\
\hline $\mathrm{SiHBr}_{3}$ & 351.0 & - & $348.0^{\mathrm{a}}, 348.6^{\mathrm{c}}, 348.05 \pm 0.4^{\mathrm{d}}$ & 81.0 & - & $80.41^{\mathrm{d}}, 80.8^{\mathrm{c}}$ \\
\hline $\mathrm{CH}$ & $\begin{array}{c}177.1 \\
(182.9)\end{array}$ & $\begin{array}{c}177.0 \\
(182.7)\end{array}$ & $183.04^{\mathrm{a}-\mathrm{b}, \mathrm{d}}$ & $\begin{array}{l}29.1 \\
(29.1)\end{array}$ & $\begin{array}{c}29.1 \\
(29.1)\end{array}$ & $29.175^{\mathrm{b}}, 29.171^{\mathrm{d}}$ \\
\hline $\mathrm{CF}$ & $\begin{array}{c}207.2 \\
(212.8)\end{array}$ & $\begin{array}{l}207.3 \\
(212.9)\end{array}$ & $213.03^{\mathrm{a}}, 213.03 \pm 0.04^{\mathrm{b}}, 213.03 \pm 0.04^{\mathrm{d}}$ & $\begin{array}{l}29.6 \\
(29.9)\end{array}$ & $\begin{array}{l}29.7 \\
(30.0)\end{array}$ & $30.06^{\mathrm{d}}, 30.06^{\mathrm{b}}$ \\
\hline $\mathrm{CF}_{2}$ & 240.7 & 240.8 & $240.83^{\mathrm{a}}, 240.838^{\mathrm{b}}, 240.833 \pm 0.04^{\mathrm{d}}$ & 38.6 & 38.9 & $38.948^{\mathrm{d}}, 38.94^{\mathrm{b}}$ \\
\hline $\mathrm{CF}_{3}$ & 264.5 & 264.7 & $265.08^{\mathrm{a}}, 264.562^{\mathrm{b}}, 264.5^{\mathrm{c}}, 265.082 \pm 4.2^{\mathrm{d}}$ & 49.6 & 49.9 & $49.804^{\mathrm{d}}, 49.58^{\mathrm{b}}, 49.6^{\mathrm{c}}$ \\
\hline $\mathrm{CF}_{4}$ & 261.6 & 261.8 & $261.41^{\mathrm{a}}, 261.454^{\mathrm{b}}, 261.6^{\mathrm{c}}, 261.419 \pm 0.25^{\mathrm{d}}$ & 61.2 & 61.6 & $61.054^{\mathrm{d}}, 61.05^{\mathrm{b}}, 61.1^{\mathrm{c}}$ \\
\hline $\mathrm{CHF}$ & 223.1 & 223.2 & $223.35^{\mathrm{a}}, 234.87^{\mathrm{b}}, 223.35 \pm 0.21^{\mathrm{d}}$ & 34.4 & 34.5 & $34.604^{\mathrm{d}}, 34.6^{\mathrm{b}}$ \\
\hline $\mathrm{CH}_{2} \mathrm{~F}$ & 235.4 & 236.1 & & 39.8 & 40.6 & \\
\hline $\mathrm{CH}_{3} \mathrm{~F}$ & 222.4 & 222.6 & $222.84^{\mathrm{a}}, 222.822^{\mathrm{b}}, 222.9^{\mathrm{c}}, 222.843^{\mathrm{d}}$ & 37.0 & 37.3 & $37.5^{\mathrm{d}}, 37.5^{\mathrm{b}}, 37.5^{\mathrm{c}}$ \\
\hline $\mathrm{CHF}_{2}$ & 255.9 & 256.1 & & 41.8 & 42.1 & \\
\hline $\mathrm{CH}_{2} \mathrm{~F}_{2}$ & 246.4 & 246.6 & $246.7^{\mathrm{a}}, 246.707^{\mathrm{b}}, 246.7^{\mathrm{c}}, 246.698 \pm 0.04^{\mathrm{d}}$ & 42.4 & 42.8 & $42.859^{\mathrm{d}}, 42.88^{\mathrm{b}}, 42.9^{\mathrm{c}}$ \\
\hline $\mathrm{CHF}_{3}$ & 259.5 & 259.8 & $259.65^{\mathrm{a}}, 259.674^{\mathrm{b}}, 259.7^{\mathrm{c}}, 259.657 \pm 0.34^{\mathrm{d}}$ & 50.7 & 51.2 & $51.04^{\mathrm{d}}, 51.07^{\mathrm{b}}, 51^{\mathrm{c}}$ \\
\hline $\mathrm{CCl}$ & $\begin{array}{c}219.2 \\
(224.5)\end{array}$ & $\begin{array}{l}219.0 \\
(224.3)\end{array}$ & $224.52^{\mathrm{a}}, 224.553^{\mathrm{b}}, 224.52 \pm 0.4^{\mathrm{d}}$ & $\begin{array}{c}31.5 \\
(32.3)\end{array}$ & $\begin{array}{c}31.5 \\
(32.3)\end{array}$ & $32.254^{\mathrm{d}}, 32.27^{\mathrm{b}}$ \\
\hline $\mathrm{CCl}_{2}$ & 265.2 & 265.1 & $265.33^{\mathrm{a}}, 265.346^{\mathrm{b}}, 265.346^{\mathrm{d}}$ & 46.6 & 46.7 & $46.249^{\mathrm{d}}, 46.25^{\mathrm{b}}$ \\
\hline $\mathrm{CCl}_{3}$ & 301.7 & 301.9 & $296.83^{\mathrm{a}}, 303.235^{\mathrm{b}}, 296.83 \pm 6.3^{\mathrm{d}}$ & 64.4 & 64.6 & $63.649^{\mathrm{d}}, 63.52^{\mathrm{b}}$ \\
\hline $\mathrm{CCl}_{4}$ & 310.4 & 310.7 & $309.65^{\mathrm{a}}, 309.461^{\mathrm{b}}, 309.809 \pm 0.04^{\mathrm{d}}$ & 84.0 & 84.4 & $83.401^{\mathrm{d}}, 82.89^{\mathrm{b}}, 83.3^{\mathrm{c}}$ \\
\hline $\mathrm{CHCl}$ & 234.9 & 234.7 & $234.91^{\mathrm{a}}, 234.91 \pm 0.21^{\mathrm{d}}$ & 36.8 & 36.8 & $36.737^{\mathrm{d}}$ \\
\hline $\mathrm{CH}_{2} \mathrm{Cl}$ & 261.6 & 246.9 & & 46.0 & 45.4 & \\
\hline $\mathrm{CH}_{3} \mathrm{Cl}$ & 234.2 & 234.2 & $234.36^{\mathrm{a}}, 234.392^{\mathrm{b}}, 234.6^{\mathrm{c}}, 234.367 \pm 0.42^{\mathrm{d}}$ & 40.7 & 40.7 & $40.731^{\mathrm{d}}, 40.74^{\mathrm{b}}, 40.8^{\mathrm{c}}$ \\
\hline $\mathrm{CHCl}_{2}$ & 281.0 & 281.6 & & 51.8 & 52.4 & \\
\hline $\mathrm{CH}_{2} \mathrm{Cl}_{2}$ & 270.4 & 270.4 & $270.28^{\mathrm{a}}, 270.36^{\mathrm{b}}, 270.2^{\mathrm{c}}, 270.293 \pm 0.04^{\mathrm{d}}$ & 51.2 & 51.3 & $50.896^{\mathrm{d}}, 50.95^{\mathrm{b}}, 51^{\mathrm{c}}$ \\
\hline $\mathrm{CHCl}_{3}$ & 296.1 & 296.2 & $295.61^{\mathrm{a}}, 296.353^{\mathrm{b}}, 295.7^{\mathrm{c}}, 295.62 \pm 0.04^{\mathrm{d}}$ & 66.0 & 66.2 & $65.383^{\mathrm{d}}, 66.85^{\mathrm{b}}, 65.7^{\mathrm{c}}$ \\
\hline $\mathrm{CBr}$ & $\begin{array}{c}230.8 \\
(233.4)\end{array}$ & - & $233.47^{\mathrm{a}}, 233.45^{\mathrm{d}}$ & $\begin{array}{c}32.5 \\
(36.1)\end{array}$ & - & $36.041^{\mathrm{d}}$ \\
\hline $\mathrm{CBr}_{2}$ & 288.8 & - & & 49.4 & - & \\
\hline $\mathrm{CBr}_{3}$ & 337.4 & - & & 69.6 & - & \\
\hline $\mathrm{CBr}_{4}$ & 358.4 & - & $358^{\mathrm{a}}, 358.057^{\mathrm{b}}, 358.1^{\mathrm{c}}, 358.095^{\mathrm{d}}$ & 91.9 & - & $91.18^{\mathrm{d}}, 90.96^{\mathrm{b}}, 91.2^{\mathrm{c}}$ \\
\hline $\mathrm{CHBr}$ & 246.5 & - & & 38.0 & - & \\
\hline $\mathrm{CH}_{2} \mathrm{Br}$ & 265.0 & - & & 46.9 & - & \\
\hline $\mathrm{CH}_{3} \mathrm{Br}$ & 245.8 & - & $245.913^{\mathrm{b}}, 246.4^{\mathrm{c}}$ & 42.4 & - & $42.45^{\mathrm{b}}, 42.4^{\mathrm{c}}$ \\
\hline $\mathrm{CHBr}_{2}$ & 304.2 & - & & 54.9 & - & \\
\hline $\mathrm{CH}_{2} \mathrm{Br}_{2}$ & 293.7 & - & $293.426^{\mathrm{b}}, 293.2^{\mathrm{c}}$ & 54.7 & - & $54.58^{\mathrm{b}}, 54.7^{\mathrm{c}}$ \\
\hline $\mathrm{CHBr}_{3}$ & 331.6 & - & $330.669^{b}, 330.9^{c}$ & 71.6 & - & $70.99^{\mathrm{b}}, 71.2^{\mathrm{c}}$ \\
\hline
\end{tabular}

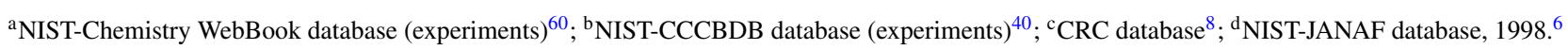


the computational data from literature, ${ }^{25-32}$ which confirm reliability of our calculation results for the $\mathrm{Si}-\mathrm{H}-\mathrm{X}$ and $\mathrm{C}-\mathrm{H}-\mathrm{X}$ compounds for $\mathrm{F}$ and $\mathrm{Cl}$. Despite lacking in scalar relativistic effects, we believe the $\mathrm{G} 4$ to be reliable enough for predicting the properties of $\mathrm{Si}-\mathrm{H}-\mathrm{Br}$ and $\mathrm{C}-\mathrm{H}-\mathrm{Br}$, which are confirmed by the $\Delta \mathrm{H}_{f}^{\circ}$ of $\mathrm{CH}_{2} \mathrm{Br}, \mathrm{CH}_{3} \mathrm{Br}, \mathrm{SiH}_{2} \mathrm{Br}$ and $\mathrm{SiH}_{3} \mathrm{Br}$ reported by Feller et al. ${ }^{25}$ and Grant and Dixon. ${ }^{32}$

Entropies and heat capacities at $298 \mathrm{~K}$.- The entropies $\left(\mathrm{S}^{298}\right)$ and heat capacities $\left(\mathrm{C}_{\mathrm{p}}{ }^{298}\right)$ at $298 \mathrm{~K}$ derived from the $\mathrm{G} 4$ and $\mathrm{W} 1 \mathrm{RO}$ unscaled harmonic frequencies are presented in Table $\mathrm{V}$ in comparison to the reported data from databases and literatures. The inclusion of low-lying electronically excited states into the electronic partition function contributes to $\mathrm{S}^{298}$ (in $\mathrm{J} \mathrm{mol}^{-1} \mathrm{~K}^{-1}$ ) with 5.3 for $\mathrm{SiH}$; 5.2 for $\mathrm{SiF}$; 4.8 for $\mathrm{SiCl} ; 3.0$ for $\mathrm{SiBr}$; 5.7 for $\mathrm{CH}$; 5.6 for $\mathrm{CF}$; 5.3 for $\mathrm{CCl}$ and 2.6 for $\mathrm{CBr}$ and to $\mathrm{C}_{\mathrm{p}}{ }^{298}$ (in $\mathrm{J} \mathrm{mol}^{-1} \mathrm{~K}^{-1}$ ) with 0.88 for $\mathrm{SiH} ; 1.09$ for $\mathrm{SiF}$; 1.63 for $\mathrm{SiCl} ; 3.51$ for $\mathrm{SiBr}$; 0.04 for $\mathrm{CH}$; 0.28 for $\mathrm{CF}$; 0.81 for $\mathrm{CCl}$ and 3.63 for $\mathrm{CBr}$. The effects are thus non-negligible for diatomic species.

Absolute enthalpies $\left(\Delta H^{T}\right)$, entropies $\left(\mathrm{S}^{T}\right)$ and heat capacities $\left(\mathrm{C}_{\mathrm{p}}{ }^{T}\right)$ at 298-2500 K.- Coefficients of 7-term NASA polynomials derived from the $\Delta \mathrm{H}^{\mathrm{T}}, \mathrm{S}^{\mathrm{T}}$ and $\mathrm{C}_{\mathrm{p}}^{\mathrm{T}}$ are provided in supplementary materials. The SOC effects are included in the $\Delta \mathrm{H}^{\mathrm{T}}, \mathrm{S}^{\mathrm{T}}$ and $\mathrm{C}_{\mathrm{p}}^{\mathrm{T}}$ for all diatomic cases. The fitting has constrained the low (high) temperature branch to reproduce the exact theoretical data at $298 \mathrm{~K}(1000 \mathrm{~K})$, and thus allows creations of small discontinuities at $1000 \mathrm{~K}$, which are less than $\sim 0.005 \mathrm{~kJ} \cdot \mathrm{mol}^{-1}$ for $\Delta \mathrm{H}^{\mathrm{T}}$ and less than $\sim 0.03 \mathrm{~J} \cdot \mathrm{mol}^{-1} \cdot \mathrm{K}^{-1}$ for $\mathrm{S}^{\mathrm{T}}$ and $\mathrm{C}_{\mathrm{p}}^{\mathrm{T}}$ for both the $\mathrm{G} 4$ and $\mathrm{W} 1 \mathrm{RO}$.

\section{Conclusions}

Thermochemical properties of the $\mathrm{Si}-\mathrm{H}-\mathrm{X}$ and $\mathrm{C}-\mathrm{H}-\mathrm{X}$ systems where $\mathrm{X}$ being $\mathrm{F}, \mathrm{Cl}$ and $\mathrm{Br}$ have been calculated using the Gaussian 4 theory (G4) and Weizman-1 theory as modified by Barnes et al. 2009 (W1RO). The calculated atomization energies were used to obtain enthalpies of formation for the complete set of molecules $(2 \times 3 \times 10$ $=60$ species). The effects of low-lying electronically excited states due to spin orbit couplings (SOCs) were included for all atoms and diatomic species by mean of electronic partition function derived from the experimental or computational energy splittings. The enthalpies of formation, entropies and heat capacities were observed to be reliable in comparison to the literature. The thermochemical properties for the temperature range of $298-2500 \mathrm{~K}$ in the form of 7-coefficient NASA polynomials are provided as supplementary materials.

\section{Acknowledgment}

Financial support from the Swedish Foundation for Strategic Research and computational resources from the Swedish National Infrastructure for Computing (SNIC) and the National Supercomputer Centre (NSC) are gratefully acknowledged.

\section{References}

1. J. Millan, P. Godignon, X. Perpina, A. Perez-Tomas, and J. Rebollo, IEEE Trans. Power Electron., 29, 2155 (2014).

2. T. Rana, M. V. S. Chandrashekhar, and T. S. Sudarshan, Phys. Status Solidi A, 209 2455 (2012).

3. T. S. Sudarshan, T. Rana, H. Song, and M. V. S. Chandrashekhar, ECS J. Solid State Sci. Technol., 2, N3079 (2013).

4. H. Pedersen, S. Leone, O. Kordina, A. Henry, S. Nishizawa, Y. Koshka, and E. Janzén, Chem. Rev., 112, 2434 (2012).

5. M. Yazdanfar, Ö. Danielsson, E. Kalered, P. Sukkaew, O. Kordina, D. Nilsson, I. G. Ivanov, L. Ojamäe, E. Janzén, and H. Pedersen, Chem. Mater, 27, 793 (2015).

6. M. W. J. Chase, NIST-JANAF Thermochemical Tables, Fourth Edition, Monograph 9 (Part I and Part II) (1998)

7. E. Goos, A. Burcat, and B. Ruscic, "Extended Third Millennium Ideal Gas and Condensed Phase Thermochemical Database for Combustion with Updates from Active Thermochemical Tables" Update of "Third Millennium Ideal Gas and Condensed Phase Thermochemical Database for Combustion with Updates from Active Thermochemical Tables, A. Burcat and B. Ruscic, Report ANL 05/20 and TAE 960 Technion-IIT, Aerospace Engineering, and Argonne National Laboratory, Chemistry Division, September 2005. (<http://burcat.technion.ac.il/dir>, 2015).
8. W. M. Haynes, editor, CRC Handbook of Chemistry and Physics, 95th Edition (Internet Version 2015) (CRC Press/Taylor and Francis, Boca Raton, FL, 2015).

9. R. Walsh, J. Chem. Soc. Faraday Trans. 1 Phys. Chem. Condens. Phases, 79, 2233 (1983).

10. J. C. Poutsma, J. A. Paulino, and R. R. Squires, J. Phys. Chem. A, 101, 5327 (1997).

11. M. Born, S. Ingemann, and N. M. M. Nibbering, Int. J. Mass Spectrom., 194, 103 (2000).

12. N. S. Shuman, L. Y. Zhao, M. Boles, T. Baer, and B. Sztáray, J. Phys. Chem. A, 112, 10533 (2008).

13. N. S. Shuman, A. P. Spencer, and T. Baer, J. Phys. Chem. A, 113, 9458 (2009).

14. Z. Tian and S. R. Kass, Int. J. Mass Spectrom., 267, 288 (2007).

15. E. R. Fisher, B. L. Kickel, and P. B. Armentrout, J. Phys. Chem., 97, 10204 (1993)

16. D. L. Hildenbrand, K. H. Lau, and A. Sanjurjo, J. Phys. Chem. A, 107, 5448 (2003).

17. B. P. Prascher, R. M. Lucente-Schultz, and A. K. Wilson, Chem. Phys., 359, 1 (2009).

18. L. Wang and Y.-L. He, Int. J. Mass Spectrom., 276, 56 (2008).

19. A. Burcat, L. Khachatryan, and B. Dellinger, Int. J. Chem. Kinet., 41, 113 (2009).

20. J. Csontos, Z. Rolik, S. Das, and M. Kállay, J. Phys. Chem. A, 114, 13093 (2010).

21. A. C. Olleta and S. I. Lane, Phys. Chem. Chem. Phys., 3, 811 (2001).

22. M. Oren, M. A. Iron, A. Burcat, and J. M. L. Martin, J. Phys. Chem. A, 108, 7752 (2004).

23. S. J. Paddison and E. Tschuikow-Roux, J. Phys. Chem. A, 102, 6191 (1998)

24. M. Schwartz, L. R. Peebles, R. J. Berry, and P. Marshall, J. Chem. Phys., 118, 557 (2003).

25. D. Feller, K. A. Peterson, W. A. de Jong, and D. A. Dixon, J. Chem. Phys., 118, 3510 (2003).

26. D. Feller, K. A. Peterson, and D. A. Dixon, J. Chem. Phys., 129, 204105 (2008)

27. M. Vasiliu, D. J. Grant, D. Feller, and D. A. Dixon, J. Phys. Chem. A, 116, 3717 (2012).

28. D. Feller and D. A. Dixon, J. Phys. Chem. A, 103, 6413 (1999).

29. W. Klopper, B. Ruscic, D. P. Tew, F. A. Bischoff, and S. Wolfsegger, Chem. Phys., 356, 14 (2009).

30. A. Karton, S. Daon, and J. M. L. Martin, Chem. Phys. Lett., 510, 165 (2011).

31. A. Tajti, P. G. Szalay, A. G. Császár, M. Kállay, J. Gauss, E. F. Valeev, B. A. Flowers, J. Vázquez, and J. F. Stanton, J. Chem. Phys., 121, 11599 (2004).

32. D. J. Grant and D. A. Dixon, J. Phys. Chem. A, 113, 3656 (2009).

33. L. A. Curtiss, P. C. Redfern, and K. Raghavachari, J. Chem. Phys., 126, 084108 (2007).

34. J. M. L. Martin and G. de Oliveira, J. Chem. Phys., 111, 1843 (1999).

35. S. Parthiban and J. M. L. Martin, J. Chem. Phys., 114, 6014 (2001).

36. E. C. Barnes, G. A. Petersson, J. A. Montgomery, M. J. Frisch, and J. M. L. Martin, J. Chem. Theory Comput., 5, 2687 (2009).

37. M. J. Frisch, G. W. Trucks, H. B. Schlegel, G. E. Scuseria, M. A. Robb, J. R. Cheeseman, G. Scalmani, V. Barone, B. Mennucci, G. A. Petersson, H. Nakatsuji, M. Caricato, X. Li, H. P. Hratchian, A. F. Izmaylov, J. Bloino, G. Zheng, J. L. Sonnenberg, M. Hada, M. Ehara, K. Toyota, R. Fukuda, J. Hasegawa, M. Ishida, T. Nakajima, Y. Honda, O. Kitao, H. Nakai, T. Vreven, J. A. Montgomery, Jr., J. E. Peralta, F. Ogliaro, M. J. Bearpark, J. Heyd, E. N. Brothers, K. N. Kudin, V. N. Staroverov, R. Kobayashi, J. Normand, K. Raghavachari, A. P. Rendell, J. C. Burant, S. S. Iyengar, J. Tomasi, M. Cossi, N. Rega, N. J. Millam, M. Klene, J. E. Knox, J. B. Cross, V. Bakken, C. Adamo, J. Jaramillo, R. Gomperts, R. E. Stratmann, O. Yazyev, A. J. Austin, R. Cammi, C. Pomelli, J. W. Ochterski, R. L. Martin, K. Morokuma, V. G. Zakrzewski, G. A. Voth, P. Salvador, J. J. Dannenberg, S. Dapprich, A. D. Daniels, Ö. Farkas, J. B. Foresman, J. V. Ortiz, J. Cioslowski, and D. J. Fox, Gaussian 09 (Gaussian, Inc., Wallingford, CT, USA, 2009).

38. L. A. Curtiss, K. Raghavachari, P. C. Redfern, V. Rassolov, and J. A. Pople, J. Chem. Phys., 109, 7764 (1998).

39. L. A. Curtiss, P. C. Redfern, V. Rassolov, G. Kedziora, and J. A. Pople, J. Chem. Phys., 114, 9287 (2001).

40. NIST Computational Chemistry Comparison and Benchmark Database, NIST Standard Reference Database Number 101, Release 16a, August 2013, Editor: Russell D. Johnson III (http://cccbdb.nist.gov/).

41. Huber G. Herzberg, Molecular Spectra And Molecular Structure, IV. Constants Of Diatomic Molecules, K. P. Huber G. Herzberg (Van Nostrand Reinhold, New York, 1979).

42. R. N. Dixon and H. W. Kroto, Trans. Faraday Soc., 59, 1484 (1963).

43. B. A. Hess and R. J. Buenker, Chem. Phys., 101, 211 (1986).

44. F. Neese, T. Petrenko, D. Ganyushin, and G. Olbrich, Coord. Chem. Rev., 251, 288 (2007).

45. K. A. Peterson, D. Feller, and D. A. Dixon, Theor. Chem. Acc., 131, 1 (2012).

46. A. Karton and J. M. L. Martin, J. Phys. Chem. A, 111, 5936 (2007).

47. N. J. DeYonker, T. R. Cundari, and A. K. Wilson, J. Chem. Phys., 124, (2006).

48. N. J. DeYonker, T. Grimes, S. Yockel, A. Dinescu, B. Mintz, T. R. Cundari, and A. K. Wilson, J. Chem. Phys., 125, 104111 (2006).

49. N. J. DeYonker, D. S. Ho, A. K. Wilson, and T. R. Cundari, J. Phys. Chem. A, 111 10776 (2007)

50. L. A. Curtiss, P. C. Redfern, K. Raghavachari, and J. A. Pople, J. Chem. Phys., 114, 108 (2001).

51. J. W. Ochterski, G. A. Petersson, and K. B. Wiberg, J. Am. Chem. Soc., 117, 11299 (1995).

52. D. Feller, K. A. Peterson, W. A. de Jong, and D. A. Dixon, J. Chem. Phys., 118, 3510 (2003).

53. J. Deng, K. Su, X. Wang, Q. Zeng, L. Cheng, Y. Xu, and L. Zhang, Theor. Chem. Acc., 122, 1 (2008). 
54. B. Ruscic, J. V. Michael, P. C. Redfern, L. A. Curtiss, and K. Raghavachari, J. Phys. Chem. A, 102, 10889 (1998)

55. K. S. Thanthiriwatte, M. Vasiliu, S. R. Battey, Q. Lu, K. A. Peterson, L. Andrews, and D. A. Dixon, J. Phys. Chem. A, 119, 5790 (2015).

56. D. Feller, K. A. Peterson, and D. A. Dixon, Mol. Phys., 110, 2381 (2012).

57. M. E. Weber and P. B. Armentrout, J. Chem. Phys., 88, 6898 (1988).

58. J. M. L. Martin and P. R. Taylor, J. Phys. Chem. A, 103, 4427 (1999).
59. M. Farber and R. D. Srivastava, Chem. Phys. Lett, 51, 307 (1977).

60. NIST Chemistry WebBook, NIST Standard Reference Database Number 69 (Eds. P. J. Linstrom and W. G. Mallard, National Institute of Standards and Technology, Gaithersburg MD, 20899, Http://webbook.nist.gov)

61. J. L. Holmes and F. P. Lossing, J. Am. Chem. Soc., 110, 7343 (1988).

62. J. A. Seetula, J. Chem. Soc. Faraday Trans., 92, 3069 (1996). 